\title{
ROSAT PSPC observations of the outer regions of the Perseus cluster of galaxies
}

\author{
S. Ettori, A.C. Fabian and D.A. White \\ Institute of Astronomy, Madingley Road, Cambridge CB3 0HA
}

29 July 2021

\begin{abstract}
We present an analysis of four off-axis ROSAT PSPC observations of the Perseus cluster of galaxies (Abell 426). We detect the surface brightness profile to a radius of $80 \operatorname{arcmin}\left(\sim 2.4 h_{50}^{-1} \mathrm{Mpc}\right)$ from the X-ray peak. The profile is measured in various sectors and in three different energy bands. Firstly, a colour analysis highlights a slight variation of $N_{\mathrm{H}}$ over the region, and cool components in the core and in the eastern sector. We apply the $\beta$-model to the profiles from different sectors and present a solution to the, so-called, $\beta$-problem. The residuals from an azimuthally-averaged profile highlight extended emission both in the East and in the West, with estimated luminosities of about 8 and $1 \times 10^{43} \mathrm{erg} \mathrm{s}^{-1}$, respectively. We fit several models to the surface brightness profile, including the one obtained from the Navarro, Frenk and White (1995) potential. We obtain the best fit with the gas distribution described by a power law in the inner, cooling region and a $\beta$-model for the extended emission.

Through the best-fit results and the constraints from the deprojection of the surface brightness profiles, we define the radius where the overdensity inside the cluster is 200 times the critical value, $r_{200}$, at $2.7 h_{50}^{-1} \mathrm{Mpc}$. Within $2.3 h_{50}^{-1} \mathrm{Mpc}\left(0.85 r_{200}\right)$, the total mass in the Perseus cluster is $1.2 \times 10^{15} M_{\odot}$ and its gas fraction is about 30 per cent.
\end{abstract}

Key words: galaxies: individual: A426 - cooling flows - dark matter - X-ray: galaxies.

\section{INTRODUCTION}

' The Perseus cluster (Abell 426) is a Bautz-Morgan type IIIII cluster, of richness class 2 , with a cD galaxy (NGC 1275) at its centre $\left(\alpha_{2000} 3^{h} 19^{m} 46.9^{s}, \delta_{2000} 41^{\circ} 30^{\prime} 48^{\prime \prime}\right)$. The cluster is located at redshift 0.0178 (Fadda et al. 1996; 1 arcmin $30.1 \mathrm{kpc}$, using hereafter $H_{0}=50 \mathrm{~km} \mathrm{~s}^{-1} \mathrm{Mpc}^{-1}$, q0 $=0.5$ and $\Lambda=0$ ).

The Perseus cluster is the brightest cluster in the $\mathrm{X}$ ray waveband, and so has been extensively studied since the UHURU mission (Gursky et al. 1971, Forman et al. 1972). The emission from its intracluster medium (ICM) is well described by a thermal gas model with a metallicity of 40 per cent of the Solar value and a temperature of $6.3 \mathrm{keV}$ (cf. Allen et al. 1992, using the GINGA satellite). The Spartan 1 detector (Snyder et al. 1990; Kowalski et al. 1993) indicates an isothermal temperature profile, beyond the cooling flow region, out to 50 arcmin radius.

However, more recent work shows that the ICM has a complex morphology in X-rays. The $505 \mathrm{~s}$ exposure from the ROSAT All Sky Survey (Schwarz et al. 1992) has shown cooler emission (by a factor of 2.5-3 times lower with respect to the rest of the cluster) to the East, corresponding to a suspected merger (recognizable between 10 and 78 arcmin from
NGC 1275). An analysis of the moments of the X-ray surface brightness distribution from the Einstein Observatory IPC observations provides additional evidence that the cluster is not yet in a relaxed state (Mohr, Fabricant \& Geller 1993). Furthermore, analysis of the ASCA GIS data (Arnaud et al. 1994) indicates hotter regions to the North-West and South-West directions, and also a slight increases in the metal abundance. A negative linear gradient in the metallicity has also been observed in the Spartan 1 data (Kowalsky et al. 1993). Furthermore, a cooling flow with a deposition rate larger than $200 M_{\odot} \mathrm{yr}^{-1}$ is located around NGC 1275 , which has an active, radio-loud nucleus (Fabian et al. 1981, Branduardi-Raymont et al. 1981, Primini et al. 1981, Rothschild et al. 1981).

In this paper, we report on the results of surveying the cluster out to radii of $80 \operatorname{arcmin}(\sim 2.4 \mathrm{Mpc})$ using a mosaic of four off-axis, ROSAT Position Sensitive Proportional Counter (PSPC) observations.

We present in section 2 the data and analysis of the surface brightness profiles using both a deprojection technique and a detailed colour analysis. We also attempt to model the gas profile with a $\beta$-model, and discuss the different behaviour according to the regions and energy bands considered. 


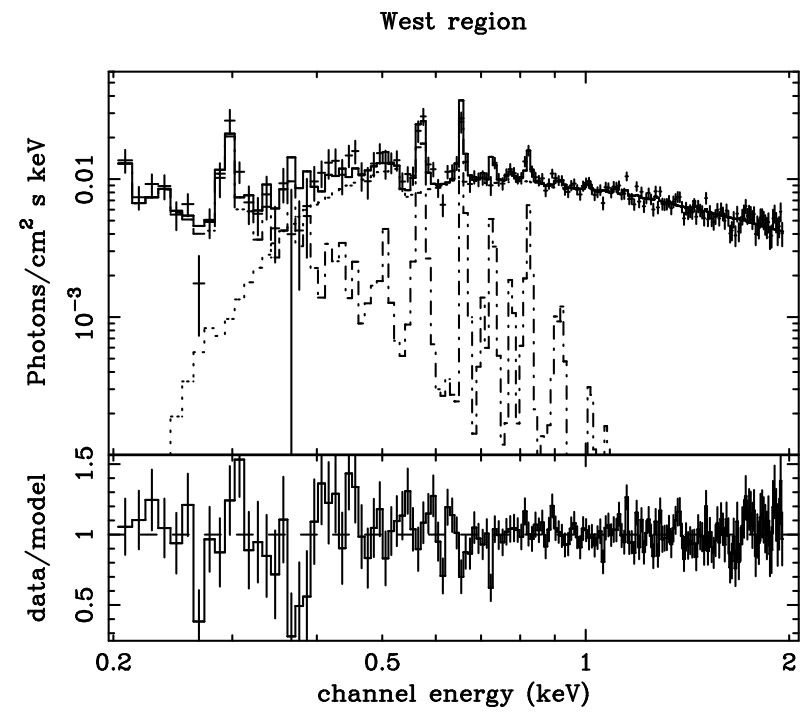

Figure 1. The unfolded spectrum is shown with its various additive components (up panel) and best-fit residuals (bottom panel). The spectrum has been made collecting the $0.2-2 \mathrm{keV}$ photons from the central $20^{\prime}$-radius region in the West region. The adopted model is the sum of a Galactic emission modelled by a Galactic Raymond-Smith thermal plasma with RaymondSmith cluster emission, with metallicity of 40 per cent of the Solar value, folded through a Galactic absorption of $12.6 \times 10^{20}$ atoms $\mathrm{cm}^{-2}$. The best-fit ( $\chi^{2}$ of 211 for 173 degrees of freedom) has parameters: $k T_{\text {Gal }}=0.22_{-0.01}^{+0.02} \mathrm{keV}, k T_{\text {cluster }}=10.19_{-2.51}^{+6.93}$ $\mathrm{keV}$.

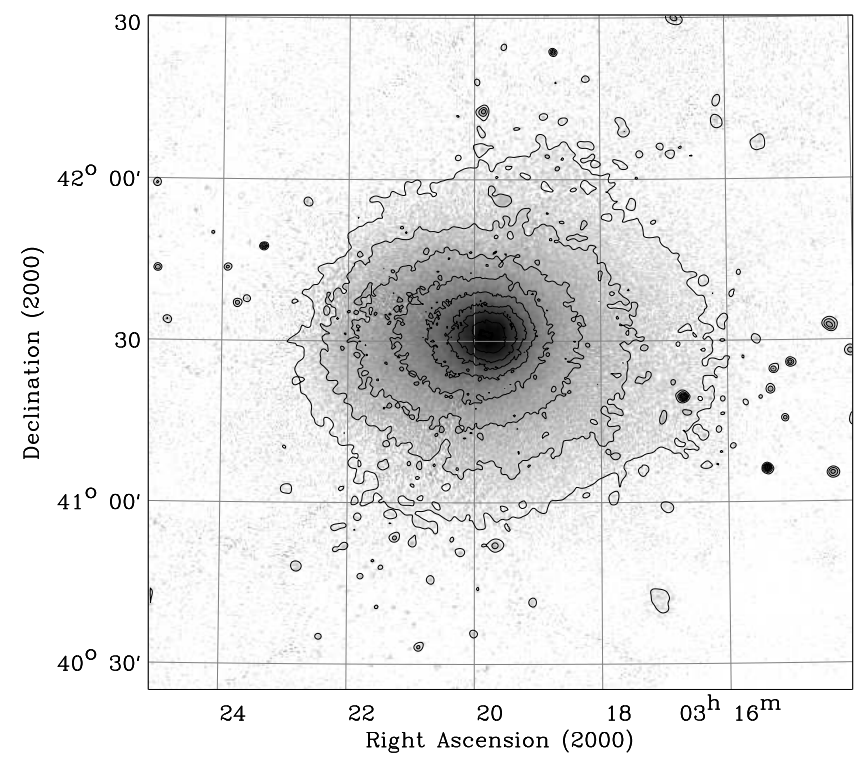

Figure 2. A mosaic of the raw data of the 4 offset observations of the Perseus Cluster, selected in the hard energy band (52-201 PI channels) and exposure-corrected, is shown here with an adaptively smoothed contours. The eight contours are equally spaced in a logarithmic scale, starting from $1.5 \times 10^{-3} \mathrm{cts} \mathrm{s}^{-1} \operatorname{arcmin}^{-2}$, which is 3 times the averaged background of the four images, and increasing by a factor of 2 to $0.192 \mathrm{cts} \mathrm{s}^{-1} \operatorname{arcmin}^{-2}$.

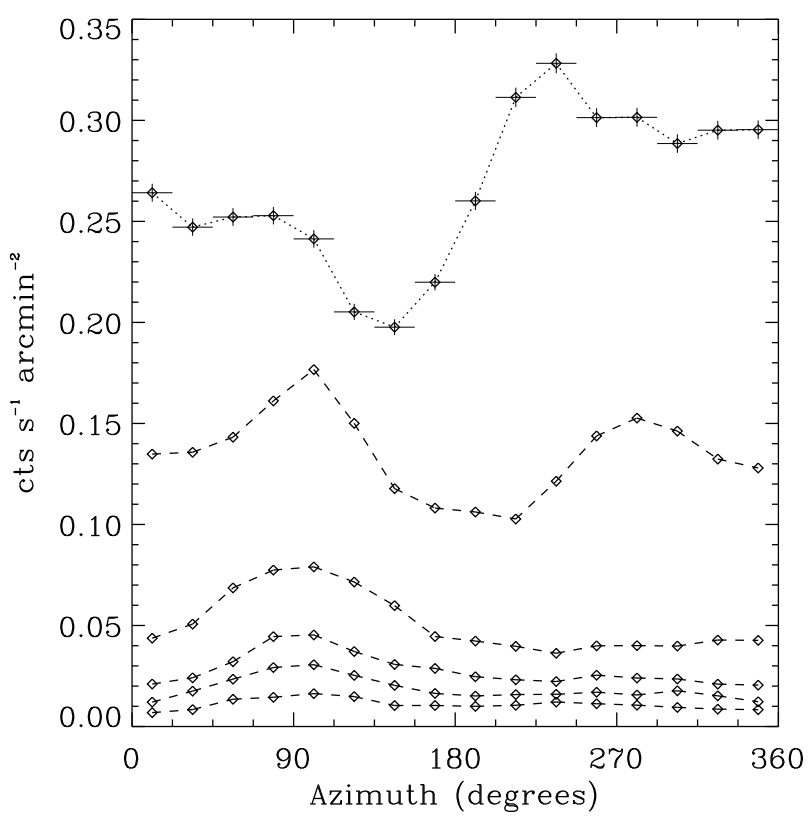

Figure 3. An azimuthal plot of the surface brightness within sectors of 22.5 , extracted around the X-ray peak in the on-axis image in steps of 4 arcmin for the region enclosed within $0<$ $r<24$ arcmin. For comparison the values in ordinate for the azimuthal profiles extracted at $r>4^{\prime}$ are multiplied by a factor of 3. Due north is defined as $0^{\circ}$ and East at $90^{\circ}$.

Our purpose is to place strict constraints on the nonluminous matter in the cluster, under the assumption of hydrostatic equilibrium, as discussed in section 3 . The main conclusions are summarized in section 4 .

\section{X-RAY DATA AND ANALYSIS}

In Table 1, we summarise the ROSAT public archive observations that we consider in our analysis.

The Perseus cluster centre is located at Galactic coordinates $\left(l, b\right.$ : $\left.150^{\circ} .58,-13^{\circ} .26\right)$. Because of its low Galactic latitude, Galactic emission provides a dominant contribution at very low energy $(\sim 0.1 \mathrm{keV})$, making (i) the corresponding energy band of little use, and (ii) any attempt to constrain fits of the spectra extracted from the off-axis regions difficult (for example, consider Fig. 11).

Following Snowden et al. (1994), we correct the images by their exposure maps in the $0.5-2.0 \mathrm{keV}$ band using the Interactive Data Language (IDL) ROSAT user-supplied libraries. A mosaic of the four off-axis observations is shown in Fig. 2, together with adaptively smoothed contours of the exposure-corrected image.

This figure clearly shows asymmetric emission along the East-West axis, which is also apparent in an azimuthal plot of the counts extracted at different radii from the central peak (cf. Fig. 3). The eastern excess (around position angle, p.a. $=90^{\circ}$ ) becomes significant at radii larger than 4 arcmin, whereas a larger contribution in emission along the WestSouthwest direction (p.a. $\sim 260^{\circ}$ ) is evident between 0 and 4 arcmin, coinciding with the position given by Schwarz et 

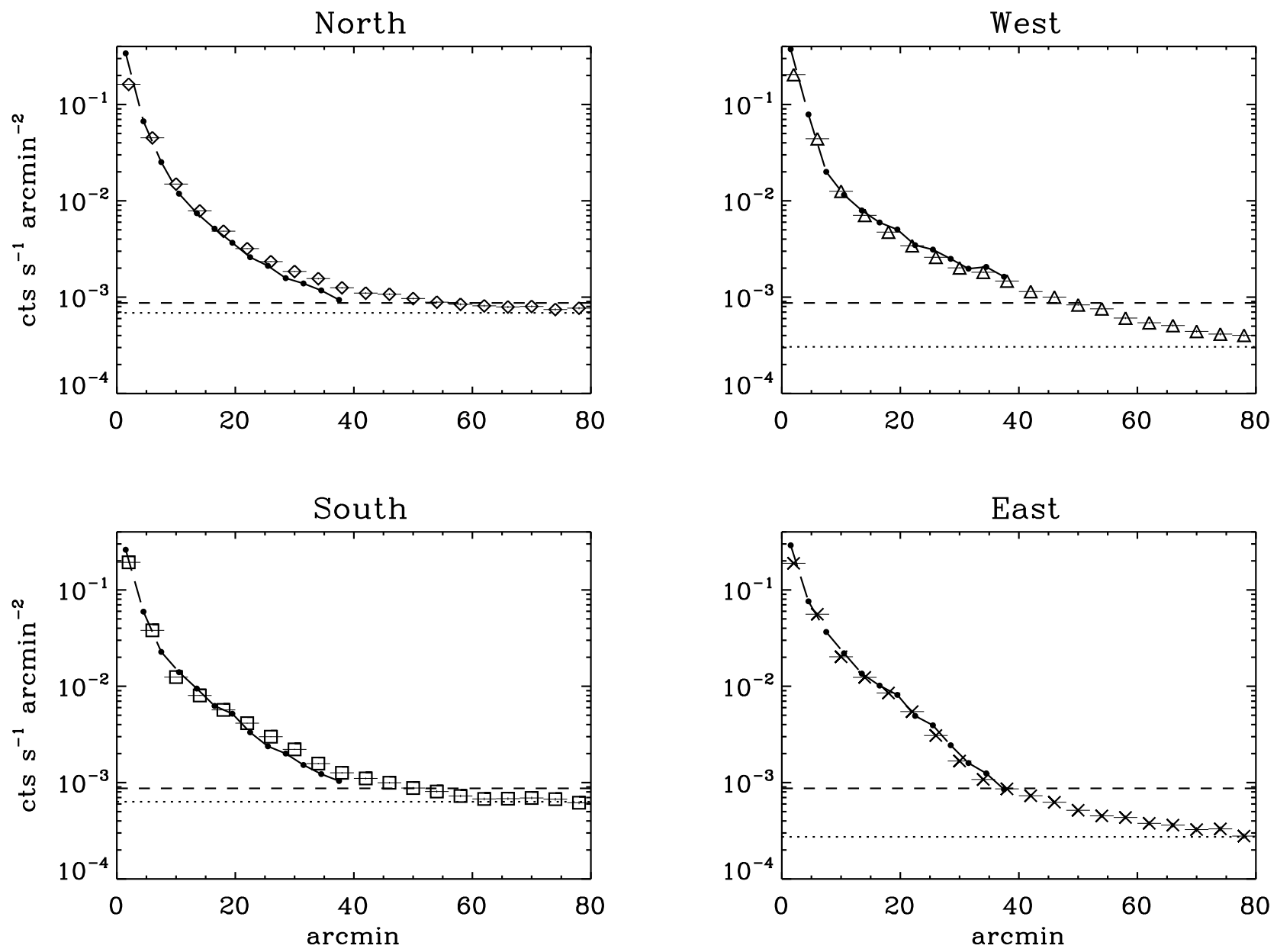

Figure 5. The surface brightness profiles in bins of 4 arcmin ware extracted from the corresponding off-axis image and exposure-corrected for the counts in the $0.5-2.0 \mathrm{keV}$ band. The dotted line indicates the background value as estimated in the $45^{\circ}$-sector opposite to the peak of the X-ray emission. The over-plotted points are the surface brightness profiles extracted, in bins of width of 3 arcmin, from the respective sectors of the on-axis image. In this case, the background (dashed line) has been estimated 40 arcmin from the X-ray peak in emission. The statistical error in each bin of the surface brightness profile is smaller than the dimension of the point symbol adopted.

Table 1. ROSAT observation summary

\begin{tabular}{|c|c|c|c|c|c|c|}
\hline Seq. Id. & Date & $\operatorname{Exp}(\mathrm{s})$ & $\alpha_{2000}$ & $\delta_{2000}$ & Region & $\mathrm{N}_{\mathrm{H}, 20}^{\ddagger}$ \\
\hline \multirow[t]{2}{*}{ wp800186 } & $199202 \mathrm{Feb}$ & 4787 & $\begin{array}{l}\text { ON-axis } \\
3^{\mathrm{h}} 19^{\mathrm{m}} 48.0\end{array}$ & $+41^{\circ} 30^{\prime} 36^{\prime \prime}$ & Centre & 14.9 \\
\hline & & & OFF-axis & & & \\
\hline rp800035 & 199118 Aug & 23669 & $3^{\mathrm{h}} 18^{\mathrm{m}} 28^{\mathrm{s}} .7$ & $+42^{\circ} 18^{\prime} 36^{\prime \prime}$ & North & 15.2 \\
\hline rp800032 & 199102 Sep & 29640 & $3^{\mathrm{h}} 15^{\mathrm{m}} 33.6$ & $+41^{\circ} 16^{\prime} 48^{\prime \prime}$ & West & 12.6 \\
\hline rp800034 & 199119 Aug & 23348 & $3^{\mathrm{h}} 21^{\mathrm{m}} 09.6$ & $+40^{\circ} 44^{\prime} 24^{\prime \prime}$ & South & 14.3 \\
\hline rp800033 & $199109 \mathrm{Feb}$ & 14566 & $3^{\mathrm{h}} 24^{\mathrm{m}} 02^{\mathrm{s}} \cdot 4$ & $+41^{\circ} 46^{\prime} 48^{\prime \prime}$ & East & 17.0 \\
\hline
\end{tabular}

$\ddagger$ This value (in units of $10^{20}$ atoms $\mathrm{cm}^{-2}$ ) is the average of the HI column density, calculated by interpolating the $(\alpha, \delta)$ coordinates from the $1^{\circ}$ grid maps by Dickey \& Lockman 1990. 


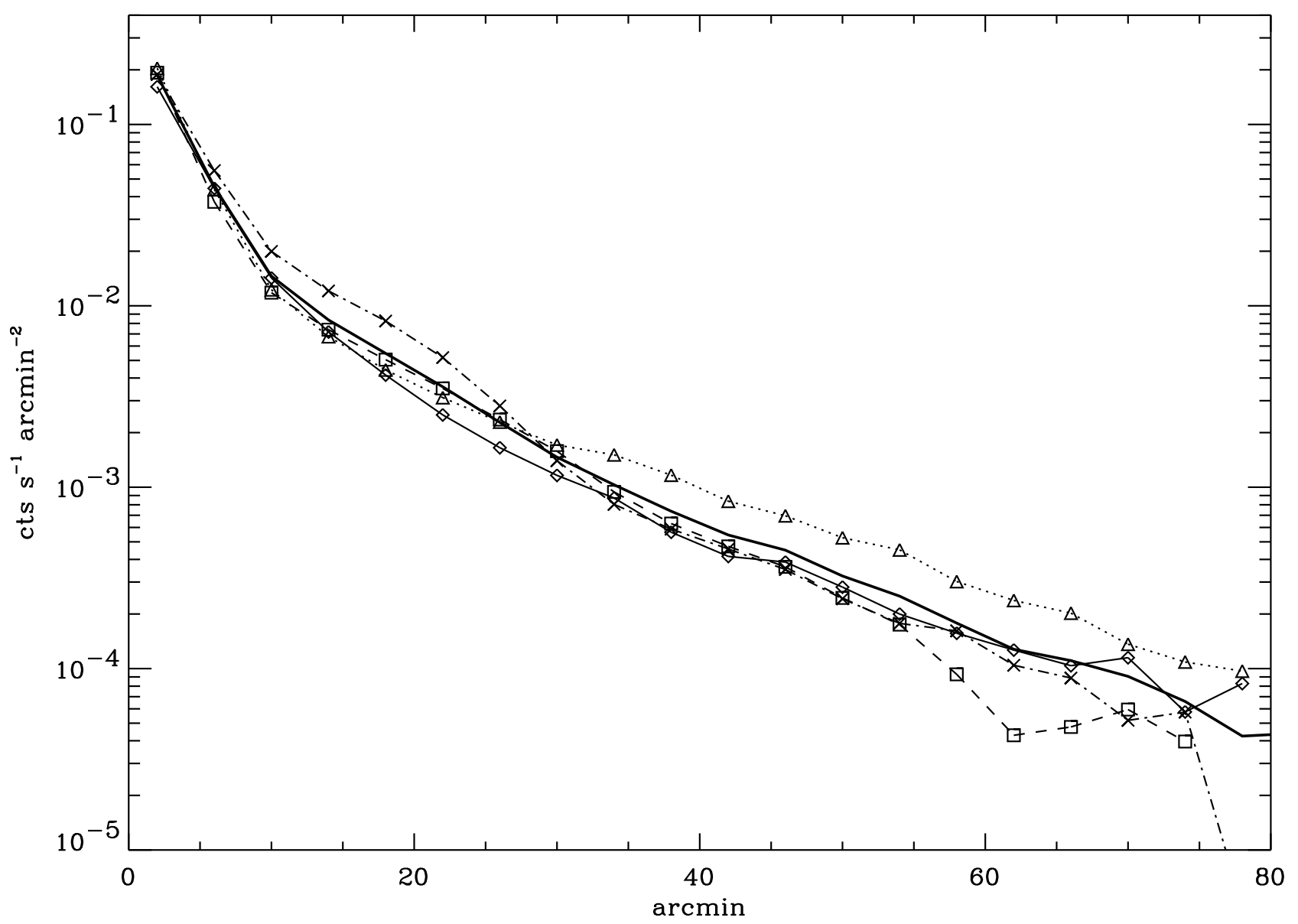

Figure 6. Here all the background-subtracted off-axis surface brightness profiles, selected in the $0.5-2.0$ keV band, are plotted together (using the corresponding symbol from Fig. 6) with their average (Centre-off profile; thick solid line).

al. (1992; cf. their Fig. 2). The latter enhancement is also seen in a wavelet analysis of the on-axis ROSAT PSPC image (cf. fig. 10 in Slezak, Durret \& Gerbal 1994), and continues in the second bin $(4-8$ arcmin). This supports the idea of an asymmetric distribution in the gas, with a preferential orientation along the 'chain' of bright galaxies to the West, out to a radius of 10 arcmin (cf. the optical image of the central part of the Perseus cluster in Fig. 1 ), and with the main excess above 10 arcmin to the East. Here, we note that further support for the idea of a merger proceeding along East-West axis is provided by the higher intracluster gas temperature observed by $A S C A$ (Arnaud et al. 1994) in the North-West and South-West regions, where the effect of the shocks are expected to increase the cluster temperature (cf. the results of hydrodynamic simulations in Schindler \& Müller 1993 and Roettinger, Loken and Burns 1997).

Moreover, an elliptical-fitting analysis with the IRAF routine ellipse shows the same behaviour of the p.a. shown in Fig. 3. We measure an ellipticity that increases from $0.06 \pm 0.04$ at 4 arcmin to $0.22 \pm 0.07$ at 24 arcmin (beyond this radius the error becomes larger than 50 per cent the value). The centroid oscillates around the X-ray peak by about 2 arcmin, first slightly to the West, then to the East, and finally Westward again (cf. Fig. 3). Apart from the Perseus cluster (cf. also Mohr et al. 1993), such oscillations are also seen in other clusters (eg. A2256, Mohr et al. 1993; Centaurus cluster, Allen \& Fabian 1994; A478, White et al. 1994; consider also the studies on the behavior of the X-ray isophotes for a sample of clusters studied by Buote \& Canizares 1996). These are indicators of an on-going merger that causes a disturbance in the intracluster gas $风$. By studying the residuals of the image, once the best-fit elliptical isophotal model is subtracted, we find significant structure only if the centroids of the isophotes are fixed to the position of the X-ray peak. Allowing each isophotes centroid to vary, eliminates significant residuals. In the following analysis, we will compare our data against the fixed centroid model of the cluster.

To extract the surface brightness profiles studied in the following analysis and presented in Figs. 5 and 6 , each observation has had the region of the PSPC support ribs masked out, as well as all detected point sources. Avoiding the central 1 arcmin where the non-thermal emission contributed

\footnotetext{
* Also an optical analysis shows a strong dependence of the position of the cluster center upon limiting magnitude and galaxy morphological types (Andreon 1994 and references therein)
} 


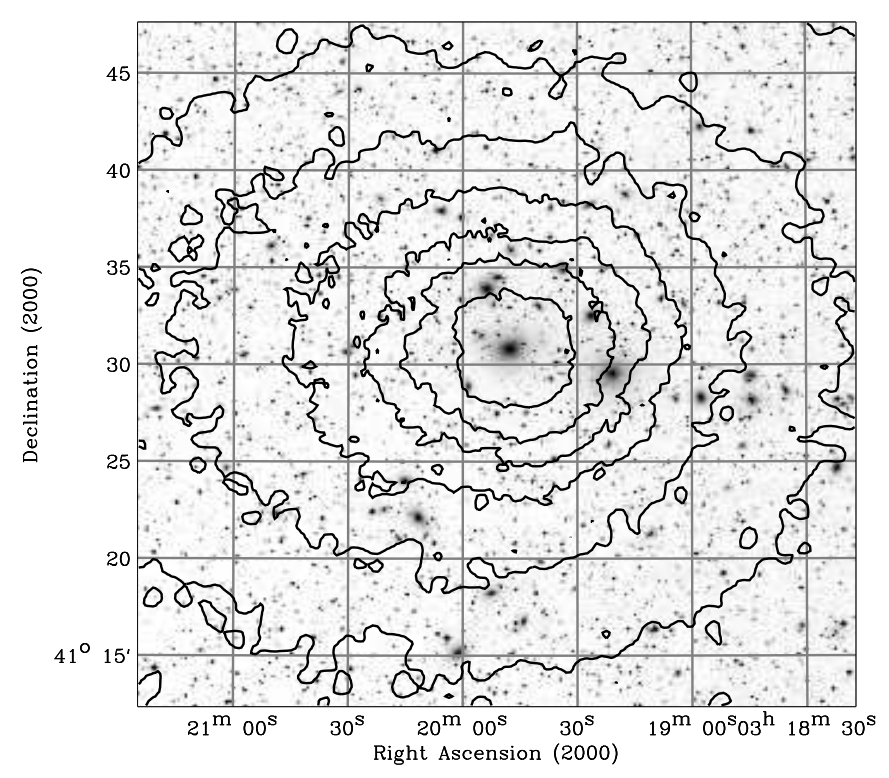

Figure 4. The adaptively smoothed contours, with the same levels as in Fig. 2, are here plotted on the Digitized Sky Survey image of the central $(30 \mathrm{arcmin})^{2}$ area of the Perseus cluster. The chain of bright galaxies is evident westwards, in a direction of NGC 1272, located at about 5 arcmin from the cD, NGC 1275.

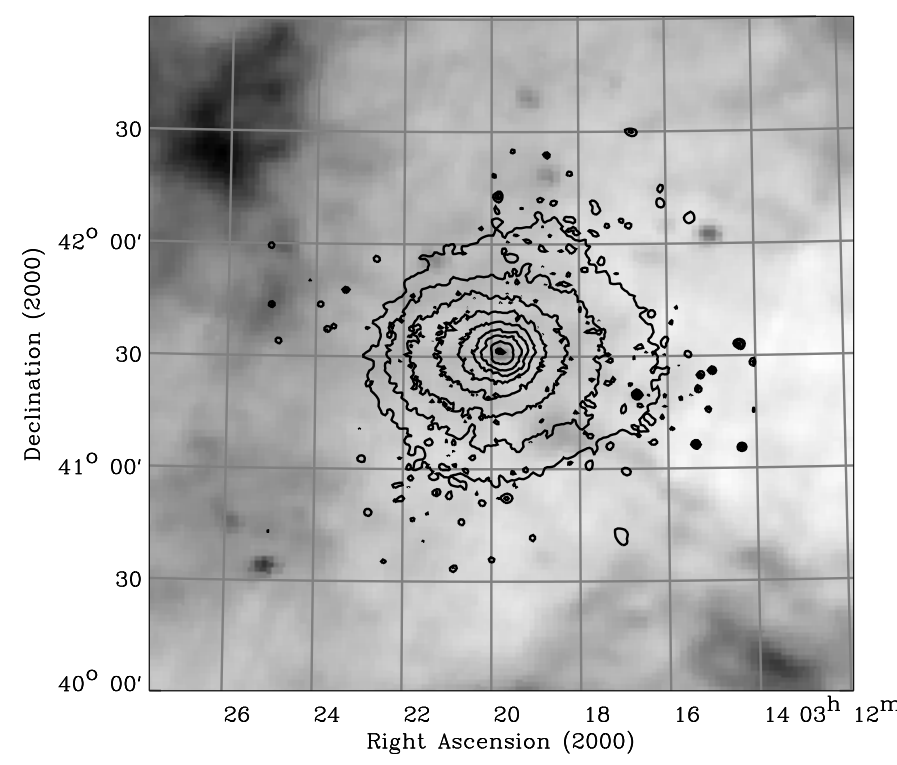

Figure 7. The eight contours of Fig. 1 are plotted on the $3^{\circ} \times$ $3^{\circ}$ IRAS 100 micron map. The IRAS data are representative of the Galactic absorption and show, on the left, the plane of the Galaxy at latitude $b \sim-12^{\circ}$. The (minimum, maximum) values in the IRAS map are $(6.1,19.2) \times 10^{6} \mathrm{Jy} \mathrm{sr}^{-1}$.

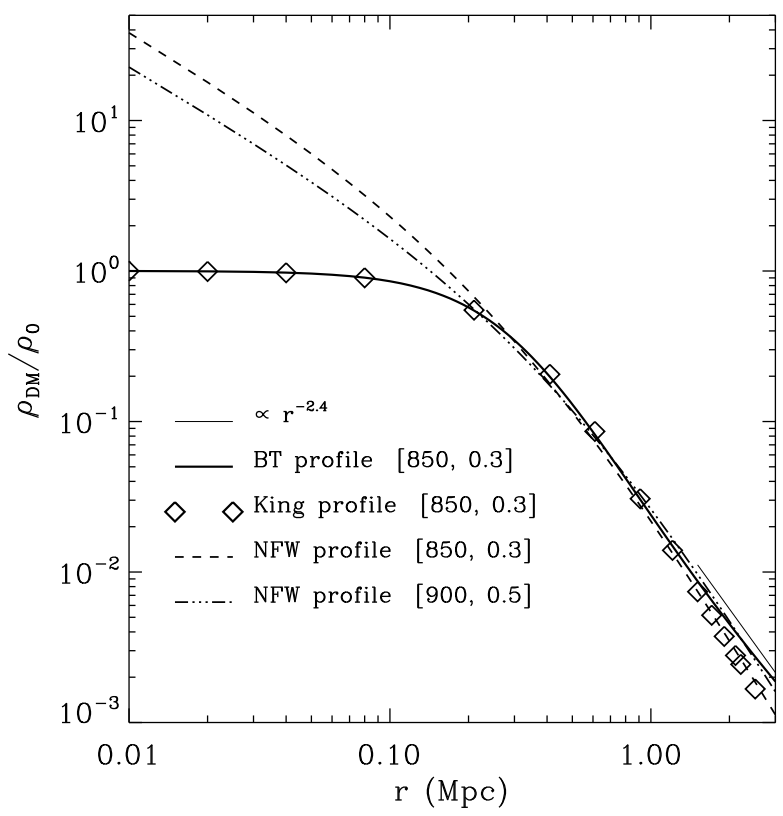

Figure 8. The Binney \& Tremaine (BT) and Navarro-FrenkWhite (NFW) dark matter profiles applied in the deprojection analysis are here compared for different input parameters, $[\sigma(\mathrm{km}$ $\left.\left.\mathrm{s}^{-1}\right), r_{\mathrm{c}}(\mathrm{Mpc})\right]$. Both of them are also compared with the King approximation to the true isothermal sphere (King 1962). All of them are normalized to the central value of the true isothermal sphere profile $\left[\rho_{0}=9 \sigma^{2} /\left(4 \pi G r_{\mathrm{c}}^{2}\right)=9.05 \times 10^{-26} \mathrm{~g} \mathrm{~cm}^{-3}\right]$. Inside the core radius, the NFW profile does not flatter like the $\mathrm{BT}$ profile. In the outer part of the region of interest $(2-3 \mathrm{Mpc})$, agreement between the two profiles is obtained by increasing the velocity dispersion and the core radius (or scale radius) in the NFW profile. Here it is also shown the dependence on $r^{-2.4}$, as recent N-body simulations of clusters of galaxies have obtained (cf. Thomas et al. 1998; the slope of this power law is the mean value estimated in the range $0.1-1 r_{180}$, where $r_{180}$ is the radius within that the average cluster density is 180 the critical one).

by NGC 1275 could be significant, the surface brightness profile has been extracted with bins of width of 3 and 4 arcmin, for the on-axis and the off-axis images, respectively. These bins widths allow smoothing over the masked circular rib at about 20 arcmin for the on-axis images, and the PointSpread-Function effect in the off-axis region of the ROSAT PSPC (cf. Fig. 5e in Hasinger et al. 1993). The profiles have been extracted from four $90^{\circ}$ sectors, North, West, South and East, respectively, of the single on-axis image. Each offaxis observation provides an azimuthally averaged surface brightness profile. We have also considered an average of these profiles (called Centre-off).

The background has been estimated in a $45^{\circ}$ sector that avoids the ribs, in a region between 40 and 45 arcmin from the centre of the image: South-East in the on-axis image, and from the opposite side of the field, from the peak of the cluster, in the offset images. The X-ray peak has been estimated by applying on the correspondent image a median filter by a square moving box with width of 5 pixels (cf. Table 2).

In Fig. $\mathrm{A}$ we show a comparison between the on-axis and the off-axis profiles. Due to the limited field-of-view of 
Table 2. X-ray centres and their separation from NGC 1275

\begin{tabular}{lccc}
\hline Region & $\alpha_{2000}$ & $\delta_{2000}$ & $\Delta \theta$ \\
\hline NGC 1275 & $3^{\mathrm{h}} 19^{\mathrm{m}} 48^{\mathrm{s}} \cdot 16$ & $+41^{\circ} 30^{\prime} 42^{\prime \prime} 1$ & - \\
On-axis & $3^{\mathrm{h}} 19^{\mathrm{m}} 46.89$ & $+41^{\circ} 30^{\prime} 48^{\prime \prime} \cdot 5$ & $0^{\mathrm{m}} 33$ \\
North & $3^{\mathrm{h}} 19^{\mathrm{m}} 46.97$ & $+41^{\circ} 32^{\prime} 11^{\prime \prime} 8$ & $1^{\mathrm{m}} 52$ \\
West & $3^{\mathrm{h}} 19^{\mathrm{m}} 42.65$ & $+41^{\circ} 30^{\prime} 23^{\prime \prime} 5$ & $1^{\mathrm{m}} 41$ \\
South & $3^{\mathrm{h}} 19^{\mathrm{m}} 43^{\mathrm{s}} .51$ & $+41^{\circ} 29^{\prime} 59^{\prime \prime} 1$ & $1^{\mathrm{m}} 37$ \\
East & $3^{\mathrm{h}} 19^{\mathrm{m}} 49.31$ & $+41^{\circ} 31^{\prime} 37^{\prime \prime} 9$ & $0^{\mathrm{m}} \cdot 97$ \\
\hline
\end{tabular}

the on-axis image and the consequential difference in estimates of the background, we only consider the off-axis surface brightness profile in the following analysis.

A comparison of the profiles shown in Fig. 6 indicates two enhancements with respect to the averaged value: (a) in the eastern sector out to 25 arcmin ; (b) beyond 30 arcmin in the West. Galactic absorption over the region (cf. Fig. 7) implies a discrepancy, in the ROSAT band flux, of up to 17 per cent between East and West, for the most and least absorbed emission respectively. This compares with the difference in the surface brightness values of a factor of about 2 at $1^{\circ}$ radius from the $\mathrm{X}$-ray centre. This underlines that the elliptical shape of the X-ray emission is not primarily due to the gradient in foreground absorption present over the region.

A further discussion about the intrinsic reality of these variations is presented in sections 2.2 and 2.3 .

\subsection{The deprojection analysis}

A deprojection analysis has been applied to the surface brightness profiles extracted from exposure-corrected image, with photons selected in the $0.5-2.0 \mathrm{keV}$ band. (The original deprojection work by Fabian et al. 1981 was on the Perseus cluster; for recent details on the deprojection technique, see White et al. 1997). Given the assumption that the observed projected cluster emission is due to the X-ray emitting gas in spherically symmetric shells, the count emissivity in each radial volume shell can be calculated analytically and compared with the predicted counts from a bremsstrahlung emission of an optically thin gas [described by a $M E K A L$ model, based on the model calculations of Mewe and Kaastra (Kaastra 1992) with Fe L calculations by Liedahl (1995)], absorbed by intervening matter $\left(N_{\mathrm{H}}\right.$ from Table 1$)$ and convolved with the response of the detector. For such an emission model, the calculated flux is proportional to the electron density, $n_{\mathrm{e}}$, and the intracluster medium temperature, $T_{\text {gas }}$, according to the relation: $n_{\mathrm{e}}^{2} f\left(T_{\text {gas }}\right)$ (where $f\left(T_{\text {gas }}\right) \stackrel{\propto}{\sim} T_{\text {gas }}^{0.5}$, for $\left.T_{\text {gas }} \gtrsim 3 \times 10^{7} \mathrm{~K}\right)$. Applying the perfect gas law and the equation of hydrostatic equilibrium to the ICM, the temperature and density profiles are obtained once a single value is known, or selected. Thus, we fix the pressure in the outermost bin to the value that allows the resulting deprojected temperature profile to match the observationally determined cluster temperature of $6.3 \mathrm{keV}$. This comes from the spectral analysis (a Raymond-Smith model plus a cooling flow component) of the data collected from the Large Area Counter on the GINGA satellite (Allen et al. 1992). The gravitational potential functional form is defined by either of two different models for the dark matter distribution: (i) the true isother- mal sphere (Binney \& Tremaine 1987); (ii) the NavarroFrenk-White dark matter profile $\left[\rho_{\mathrm{DM}}=\rho_{\mathrm{s}} x^{-1}(1+x)^{-2}\right.$; Navarro et al. 1995]. A comparison between them for different input parameters is shown in Fig. There, the $\left[\sigma, r_{\mathrm{c}}\right]$ values for the case of the NFW potential are the velocity dispersion of the dark matter under the isothermal condition 7, and the scale radius, as defined in Navarro et al. (1995).

A gravitational contribution from the $\mathrm{cD}$ galaxy NGC 1275 has also been included using a de Vaucouleurs (1948) model with a velocity dispersion of $400 \mathrm{~km} \mathrm{~s}^{-1}$ (from the optical $L-\sigma^{4}$ relation on the brightest cluster members in Malumuth \& Kirshner 1985) and an effective radius of 21 kpc (Schombert 1987).

In Fig. 9, as an example of the information obtained by deprojecting a surface brightness profile, we show the results from the off-axis observation of the North of the Perseus cluster.

The standard deviation confidence limits on the results summarized in Table 3 are estimated by perturbing the surface brightness profile 100 times, according with the Poissonian error on the counts in each radial bin. For the cooling radius, $r_{\text {cool }}$, and the mass deposition rate, $\dot{M}$, we quote the median values, with the respective 10 th and 90 th percentile limits, that come from the 100 perturbations.

The gas mass, $M_{\text {gas }}$, and the total gravitating mass, $M_{\text {tot }}$, are obtained by integrating the gas and the dark matter density, respectively, over the volume. The gas fraction, $f_{\text {gas }}$, is defined as the ratio between the total gas mass and the gravitating mass within the radius $R_{\text {out }}$, where the surface brightness is larger than its error by, at least, a factor of 3 .

The two dark matter profiles that we adopt, the true isothermal sphere and the Navarro-Frenk-White profile, give good agreement in describing the physical condition of the ICM under the hydrostatic and isothermal assumptions, apart from a systematic reduction (about 10 per cent in the averaged values) in the mass deposition rate when using a NFW dark matter profile rather than the BT profile.

However, given the low resolution that we adopt, to avoid the technical limits on the observations (i.e., masked ribs, off-axis PSF) and to ensure a good signal-to-noise ratio in the outer part of the cluster, we do not investigate further the cooling flow region, apart from emphasizing that the results are roughly consistent with each other, and also with recent work that indicates a cooling radius of $0.2 \mathrm{Mpc}$ and $\dot{M}$ of about $500 M_{\odot} \mathrm{yr}^{-1}$ (cf. Allen \& Fabian 1997, Peres et al. 1998).

\subsection{The colour analysis}

Given the low spectral resolution of the PSPC $[\Delta E / E=$ $0.43(E / 0.93 \mathrm{keV})^{-0.5} ;$ ROSAT Users' Handbook 1994 ]], we define three independent energy bands (or colours) where

$\ddagger \sigma^{2}=G M_{200} /\left(2 r_{200}\right)=50 H^{2} r_{200}^{2}$, where $M_{200}, r_{200}, H$ and $G$ are the dark matter mass at the over-density of 200, the radius at which this over-density is reached, the Hubble constant and the Gravitational constant, respectively

$\dagger$ also available at the URL heasarc.gsfc.nasa.gov/docs/rosat/ruh/handbook/handbook.html 


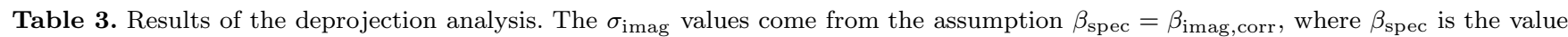

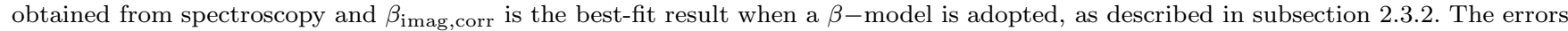
on the gas fraction, $f_{\text {gas }}$, consider the uncertainty on the temperature, $k T$, added in quadrature. Each column contains the two values obtained under the assumption that the cluster gravitational potential is described by either a true isothermal sphere (BT) or using the Navarro-Frenk-White form (NFW).

\begin{tabular}{|c|c|c|c|c|c|c|c|}
\hline Region & $\begin{array}{c}r_{\mathrm{c}} \\
\mathrm{Mpc}\end{array}$ & $\begin{array}{c}\sigma_{\mathrm{dpr}}\left(\sigma_{\text {imag }}\right) \\
\mathrm{km} \mathrm{s}^{-1}\end{array}$ & $\begin{array}{l}P_{\text {out }} / 10^{4} \\
\mathrm{~K} \mathrm{~cm}^{-3}\end{array}$ & $\begin{array}{l}R_{\text {out }} \\
\mathrm{Mpc}\end{array}$ & $\begin{array}{l}r_{\text {cool }} \\
\mathrm{Mpc}\end{array}$ & $\begin{array}{c}\dot{M} \\
M_{\odot} \mathrm{yr}^{-1}\end{array}$ & $f_{\text {gas }}$ \\
\hline & BT, NFW & BT, NFW & & & BT, NFW & BT, NFW & BT, NFW \\
\hline North & $0.25,0.35$ & $\begin{array}{l}860,860 \\
\left(896_{-33}^{+46}\right)\end{array}$ & 1.7 & 1.93 & $0.20_{-0.02}^{+0.10}, 0.20_{-0.02}^{+0.10}$ & $499_{-11}^{+29}, 474_{-11}^{+47}$ & $0.31 \pm 0.01,0.31 \pm 0.01$ \\
\hline West & $0.45,0.60$ & $\begin{array}{l}830,860 \\
\left(85 \gamma_{-21}^{+26}\right)\end{array}$ & 1.6 & 2.29 & $0.21_{-0.03}^{+0.09}, 0.20_{-0.02}^{+0.10}$ & $554_{-61}^{+19}, 487_{-30}^{+5}$ & $0.37 \pm 0.01,0.40 \pm 0.01$ \\
\hline South & $0.60,1.80$ & $\begin{array}{l}970,1150 \\
\left(1148_{-67}^{+96}\right)\end{array}$ & 2.1 & 1.69 & $0.20_{-0.02}^{+0.10}, 0.20_{-0.02}^{+0.10}$ & $489_{-45}^{+5}, 452_{-24}^{+1}$ & $0.21 \pm 0.01,0.22 \pm 0.01$ \\
\hline East & $0.50,0.70$ & $\begin{array}{l}960,1000 \\
\left(1029_{-41}^{+54}\right)\end{array}$ & 1.7 & 1.93 & $0.22_{-0.04}^{+0.09}, 0.20_{-0.02}^{+0.10}$ & $629_{-9}^{+14}, 543_{-13}^{+49}$ & $0.24 \pm 0.01,0.26 \pm 0.01$ \\
\hline Centre-off & $0.40,0.70$ & $\begin{array}{l}890,960 \\
\left(987_{-41}^{+53}\right)\end{array}$ & 1.2 & 2.29 & $0.21_{-0.02}^{+0.10}, 0.20_{-0.02}^{+0.10}$ & $519_{-17}^{+3}, 477_{-3}^{+7}$ & $0.30 \pm 0.01,0.29 \pm 0.01$ \\
\hline
\end{tabular}

the analysis will be performed: (i) a soft band, which consider photons with energy enclosed between 0.52 and 0.90 $\mathrm{keV}$ (formed by the sum of the R4 and R5 ROSAT bands; cf. table 1 in Snowden et al. 1994); (ii) a medium band, with an energy range of $0.91-1.31 \mathrm{keV}$ (R6 ROSAT band), which contains most of the Fe-L emission produced by gas at about $1 \mathrm{keV}$; (iii) a hard band (corresponding to R7) between 1.31 and $2.01 \mathrm{keV}$. These 3 bands from on-axis PSPC response curve are shown in Fig. 10.

We make use of the ratios among these colours to probe the physical properties of the Perseus cluster, once the observed ratio values are compared with the behaviour of the same ratio in a simulated cluster spectra. This kind of analysis using the ROSAT PSPC colours is also comprehensively discussed in Allen \& Fabian (1997) in a study of the cooling flows properties of a sample of clusters.

Predicted colour ratios were calculated in XSPEC (vers. 10; Arnaud 1996) using as reference model the plasma code of Mewe, Kaastra and Liedahl (MEKAL model; Kaastra 1992, Liedahl et al. 1995), with a metallicity, $Z$, of either 40 or 70 per cent the Solar abundance $Z_{\odot}$, and subjected to the photoelectric absorption provided from the model of Morrison \& McCammon (1983). We present in Fig. 11, for a wide range of ICM temperature and Galactic absorption, the theoretical ratios obtained fixing one of the parameters.

Firstly, these plots show that it appears difficult to detect any gradient in metallicity [note that the squares and the diamonds overlap]. They also show a weak dependence of the ratios upon temperatures higher than $3 \mathrm{keV}$. More meaningful are the steepening of the ratios at low temperatures, particularly in the medium/hard [(91-131 PI)/(132$201 \mathrm{PI})]$ colors ratio, and the decrease in soft/hard [ $52-90$ PI)/(132-201 PI)] when a higher column density is considered (cf. the panels with triangles and diamonds). We also note that, if $N_{\mathrm{H}}$ is assumed much lower than its true value, this effect hides the steep increase due to the presence of a lower gas temperature, particularly in the ratio where the softest band is involved.

We performed a colour analysis along the four directions (North, West, South, East) where the cluster has been observed. All the four offset observations allow us to extract the surface brightness profile, $S(r)$, out to 80 arcmin from the X-ray centre. We use bins of $[4,5,6, \ldots]$ arcmin to increase the signal-to-noise ratio at larger radii (cf. Fig. 12 ).

The background was selected in a $45^{\circ}$-sector opposite the peak of the X-ray emission and calculated as an average of the counts s ${ }^{-1} \operatorname{arcmin}^{-2}$ present between $40^{\prime}$ and $45^{\prime}$. A Poissonian error is applied to the counts of photons present in the selected region.

The errors, $\epsilon_{\mathrm{col}}$, on the colours ratio, $S_{\mathrm{col}}$, come from the propagation, in quadrature, of the $1-\sigma$ uncertainty both in each radial bin and in the background:

$S_{\mathrm{col}}=\frac{S_{1}-S_{\mathrm{bkg}, 1}}{S_{2}-S_{\mathrm{bkg}, 2}}, \quad \frac{\epsilon_{\mathrm{col}}^{2}}{S_{\mathrm{col}}^{2}}=\sum_{i=1,2} \frac{\epsilon_{i}^{2}+\epsilon_{\mathrm{bkg}, i}^{2}}{\left(S_{i}-S_{\mathrm{bkg}, i}\right)^{2}}$,

where $S$ and $\epsilon$ are the surface brightness value and the related error, respectively, the index 'bkg' denotes the background value. The sum is performed on the 2 energy bands considered. These errors increase towards the outer regions, becoming dominant at radius larger than 50 arcmin (around 60 arcmin the signal to noise ratio, $S / N$, becomes $\lesssim 3$ for bin size of $[4,5,6, \ldots]$ arcmin; cf. Fig. 12 for the dependence of $S / N$ on the bin size.).

We describe below the results of the colour analysis performed through the ratios $S_{\mathrm{col}}(r)$. Here we note that any apparent gradient has to be considered with respect to the error bars. We observe a slight dependence on the background of the colour profile in the outer region, whilst the $\sigma$ devi- 
North
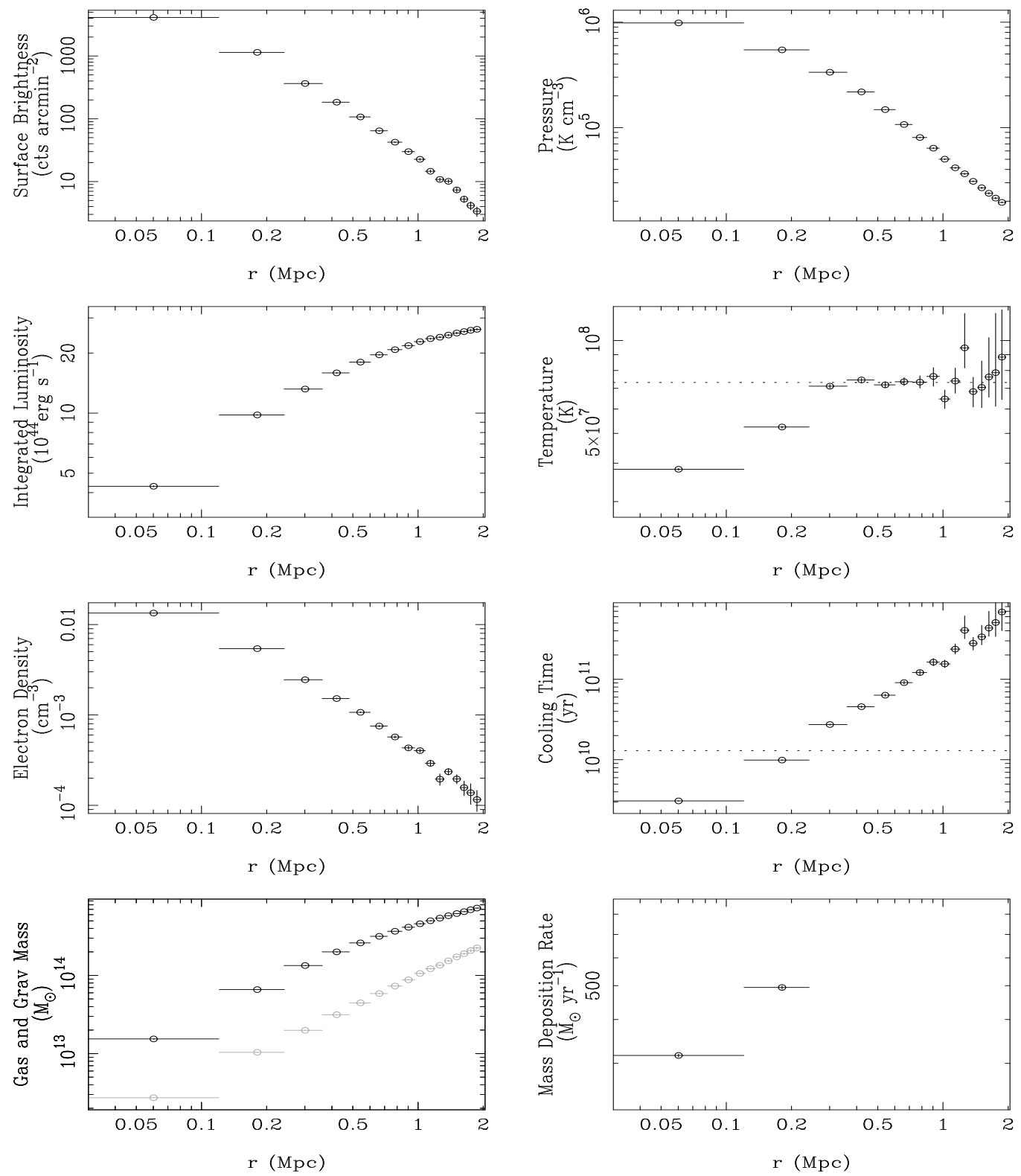

Figure 9. Plots of the deprojection results of the northward observation of the Perseus cluster, when the potential for a true isothermal sphere is applied. The dotted lines in 'Temperature' and 'Cooling Time' plots represent the adopted $T_{\text {gas }}$ of $6.3 \mathrm{keV}$ and the Hubble time, respectively. In Table 3, we list the input and output values of the deprojection analysis.

ations (showed in lower panels of Fig. 13 16) are consistent whichever background area is selected.

Our results are also compared to the conclusions from the $(2.5-10 \mathrm{keV}) /(1-2.5 \mathrm{keV})$ colour analysis on the Spartan 1 data (Snyder et al. 1990).

North The Spartan 1 results show in this direction a slight increase in the $(2.5-10 \mathrm{keV}) /(1-2.5 \mathrm{keV})$ ratio moving away from the cluster centre, that they claim is related to the observed higher Galactic absorption to the NE of Perseus (cf. Fig. 7).
In our analysis (cf. Fig. 13), we observe a $3 \sigma$ fluctuation in the core region due to the cooling component. Apart from other $2 \sigma$ fluctuations around 20 arcmin, a plasma model with $k T=6.3 \mathrm{keV}$ and $N_{\mathrm{H}}=15.2 \times 10^{20}$ atoms $\mathrm{cm}^{-2}$ is a good description of the data.

West In this region, Spartan 1 does not show any colour gradient.

A cool component ( $\lesssim 3 \mathrm{keV}$ ) is present at high significance in the core (panel b). The negative $3 \sigma$ deviation in the second bin can be explained only with an increase by a factor of about 2 in the absorption with respect to the value quoted 

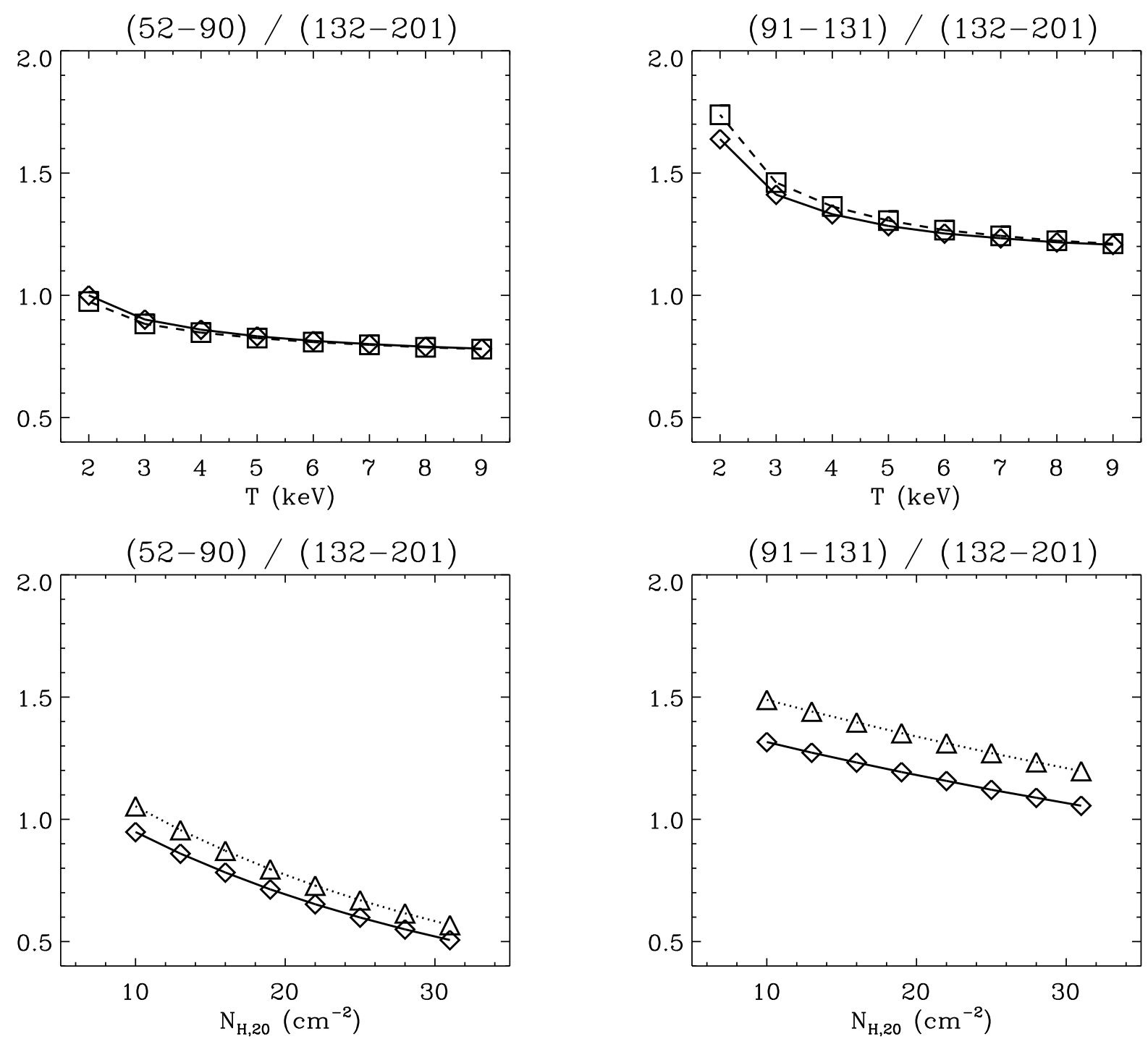

Figure 11. The diamonds connected by solid line represent the $\left(T=6.3 \mathrm{keV} ; N_{\mathrm{H}, 20}=14.9 ; Z / Z_{\odot}=0.4\right)$ case for the two different ratios considered, when either the temperature is varied between 2 and $7 \mathrm{keV}$ (upper panels) or $N_{\mathrm{H}, 20}$ is in the range 10-30 (lower panels). In the panels at the top, the squares show the (varies; 14.9; 0.7) choice. In the panels at the bottom, the triangles represent the (3.0; varies; 0.4$)$ case. These plots show that it appears difficult to detect any gradient in metallicity [note as the squares and the diamonds overlap]. They also show a weak dependence of the ratios upon temperatures higher than $3 \mathrm{keV}$, and a significant decrease when an higher column density is considered (cf. the panels with triangles and diamonds).

of $N_{\mathrm{H}, 20}=12.6$ (Fig. 14). This $3 \sigma$ deviation is still evident between 15 and 30 arcmin, where the absorption remains high (cf. also Fig. 7) and GINGA (Arnaud et al. 1994) detects clumps at temperatures of about $9 \mathrm{keV}$. Both of these effects decrease the colour ratios, even if the excess in absorption is dominant and requires roughly a $\Delta N_{\mathrm{H}, 20} \sim 3$. Moreover, between 35 and 50 arcmin, there is a drastic increase in the ratios due to the presence of a cool component, corresponding to the region around the galaxy IC310.

South Snyder et al. (1990) observe a very flat colour profile.

Again, the colour analysis underlines the cooling region within 4 arcmin and an excess in absorption within 10 arcmin of about $4 \times 10^{20} \mathrm{~cm}^{-2}$ with respect to the quoted value of $N_{\mathrm{H}, 20}=14.3$. Furthermore, we obtain consistency with a $k T=6.3 \mathrm{keV}$ above 10 arcmin, from panel (b) (Fig. 15).
East At radii greater than 25 arcmin, Snyder et al. (1990; cf. their fig. 11d) observe a softening of the cluster spectrum. With an estimated temperature of $2.5 \mathrm{keV}$, the core presents the larger deviation from the assumed isothermal model. Then, at least in the first 3 bins, we are overestimating $N_{\mathrm{H}}$ [from the temperature in panel (b), we require something more close to $\left.N_{\mathrm{H}, 20} \sim 14\right]$. We also note that the colour ratio behaviour is more consistent with a cooler ICM (Fig. 16 shows less dispersion for the solid line that represents the gas at $3 \mathrm{keV}$ ) between 20 and 50 arcmin. Here, a slight positive gradient in the colour profiles is present between the third and sixth bin (i.e. 9-39 arcmin), underlying a softening of the emission where the excess in surface brightness is detected (cf. also Fig. 3 and 6).

To summarize, we observe variations in X-ray colour 

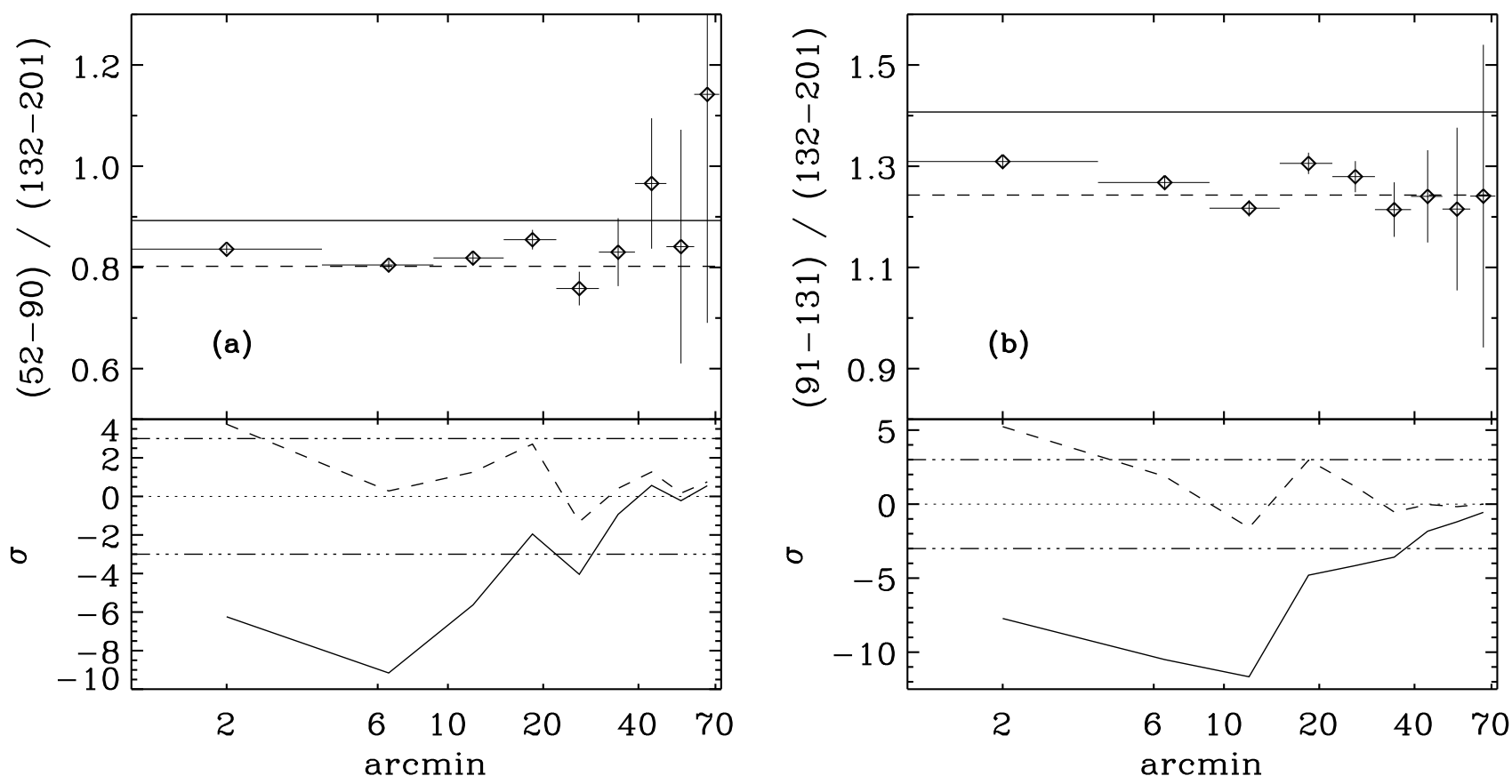

Figure 13. The colour ratios for the North region. The Galactic absorption value used is quoted in Table 1. (Upper panels) The colour ratios $S_{\mathrm{col}}(r)$ are compared with the predicted value at $3 \mathrm{keV}$ (solid line) and at $6.3 \mathrm{keV}$ (dashed line). (Lower panels) The deviation (in $\sigma$ ) from the predicted values ( $3 \mathrm{keV}$ : solid line; $6.3 \mathrm{keV}$ : dashed line) are plotted. In these panels, the dotted line shows the zero level and the dash-dot-dot line the $\pm 3 \sigma$ level.
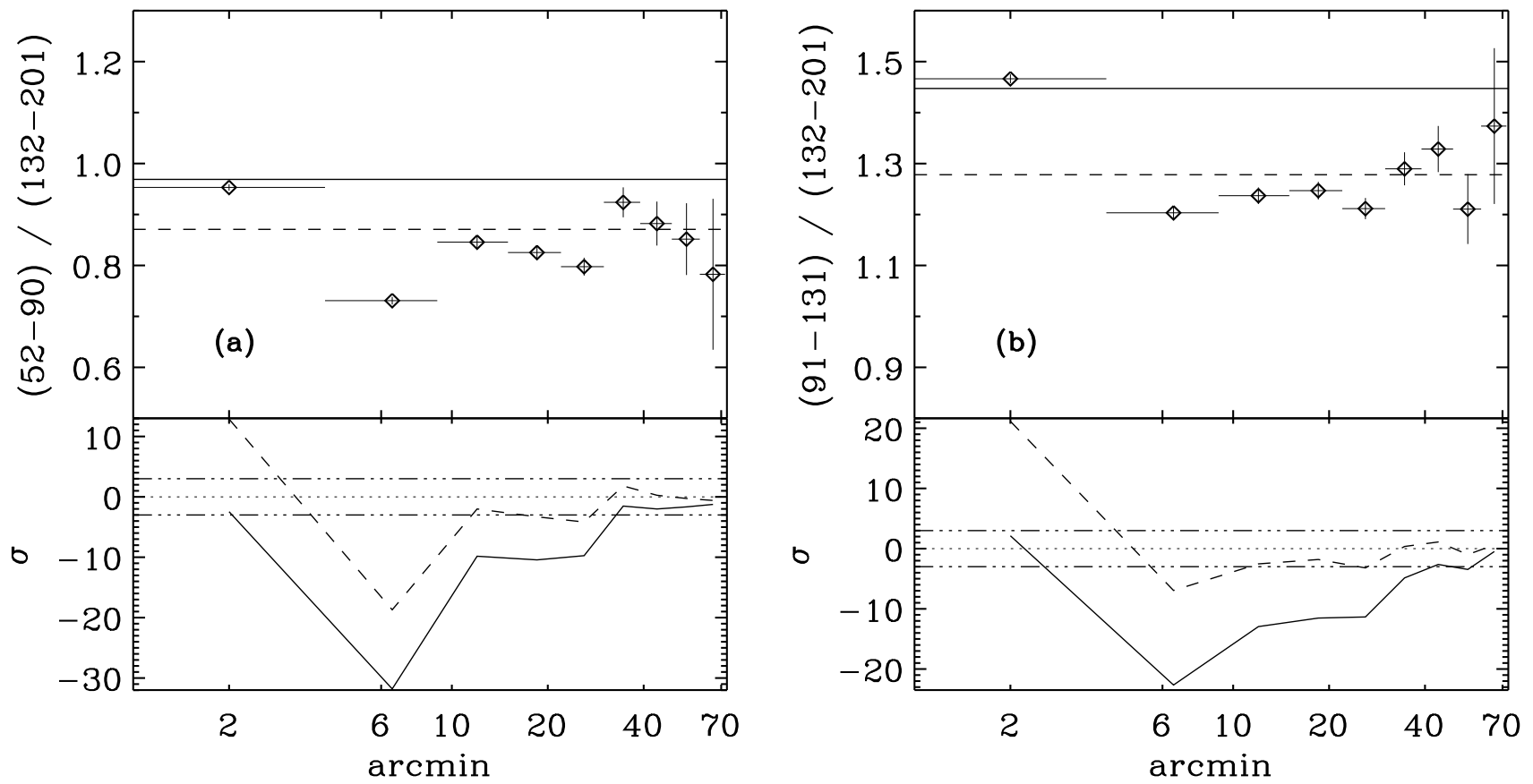

Figure 14. As Fig. 13 for the West region. 

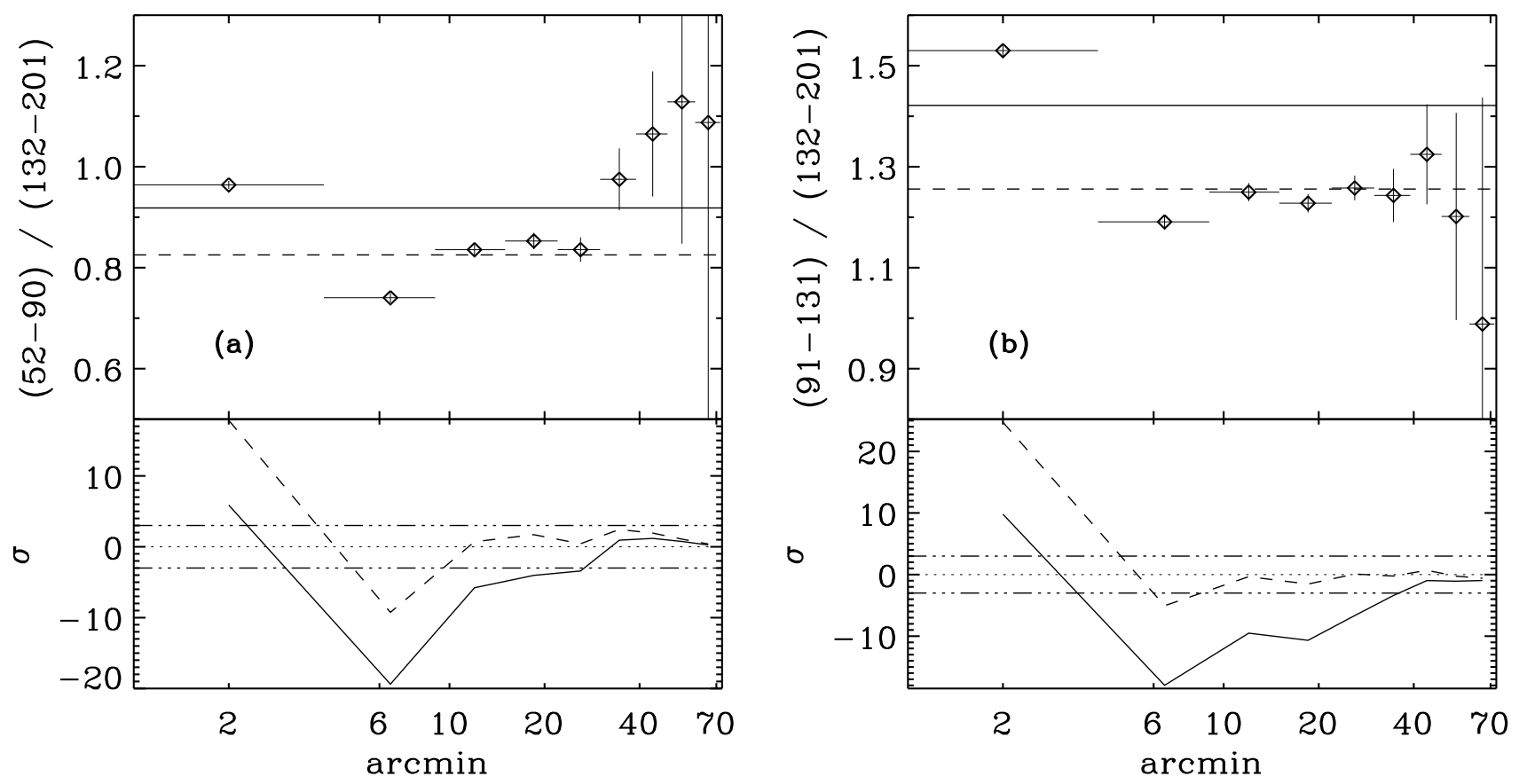

Figure 15. As Fig. 13 for the South region.
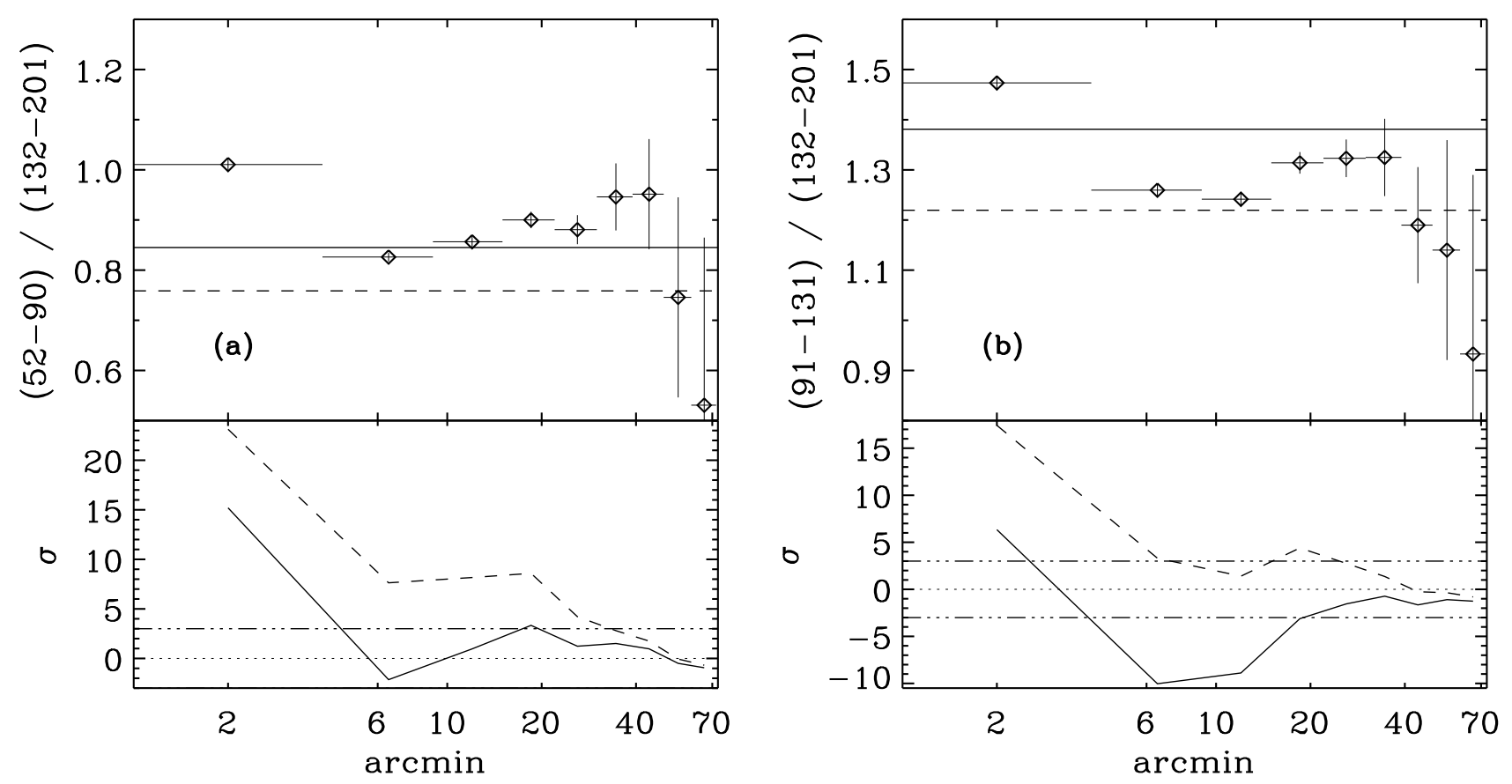

Figure 16. As Fig. 13 for the East region. 


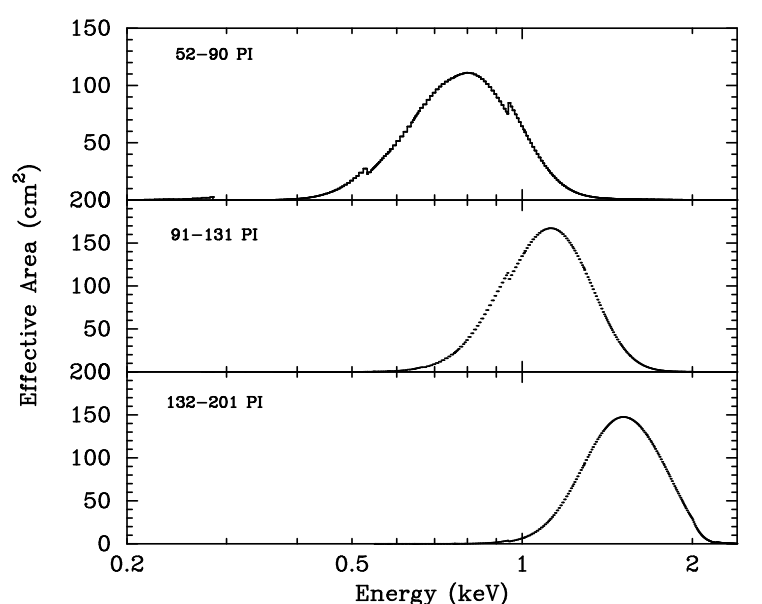

Figure 10. These are the Effective Areas of the 3 energy bands considered. They are extracted from the Redistribution Matrix File pspcb_gain2_256.rmf.

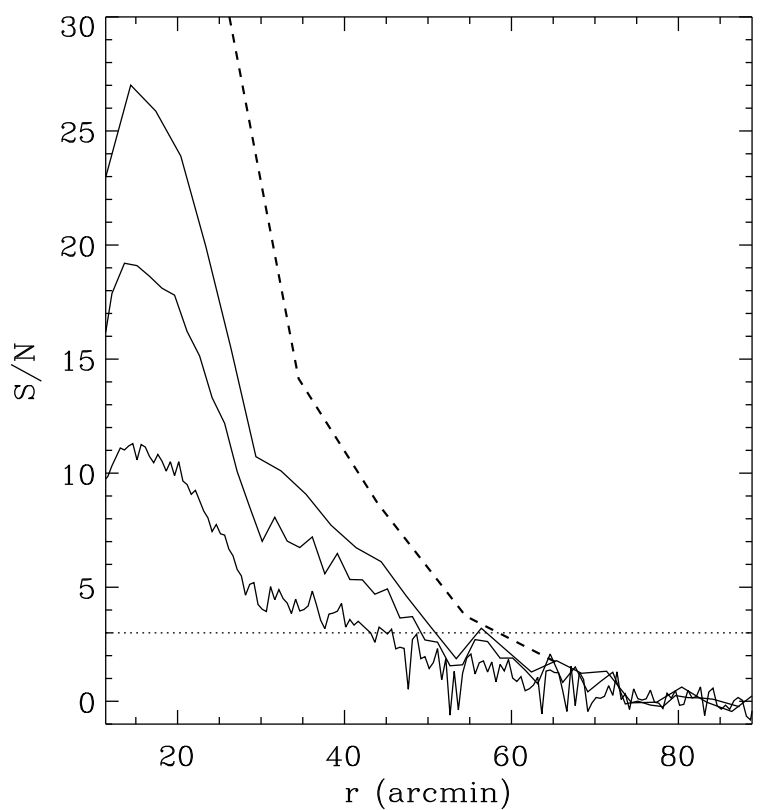

Figure 12. Signal to noise ratio for Soft/Hard band ratio in the East with bins size of 30 arcsec, 90 arcsec and 180 arcsec (upwards solid lines). The dashed line is for bins size of $[4,5,6, \ldots]$ arcmin and its maximum is about 90 in the first 2 bins. The dotted line represents the threshold of $S / N=3$.

across the four regions. There is a clear temperature decrease in the core associated with the cooling flow. At larger radii from the centre the changes are best explained by small variations in $N_{\mathrm{H}}$. There is no evidence for an overall systematic temperature gradient up to $50 \operatorname{arcmin}(\sim 1.5 \mathrm{Mpc})$.

\subsection{The $\beta$ problem}

Cavaliere \& Fusco-Femiano (1976) pointed out that the scale height for galaxies and gas in a cluster may be calculated
Table 4. Best $\beta$-model fit values from previous work. The errors are at the 90 per cent confidence level. The labels in column ref indicate: Cr97 (Cruddace et al. 1997), Sc92 (Schwarz et al. 1992), Sn90 (Snyder et al. 1990), JF84 (Jones \& Forman 1984), Br81 (Branduardi-Raymont et al. 1981); Cr97 and Sn90 apply two different method on the same data from the Spartan I: Cr97 bin the spatial and the spectral counts distribution and fit a three-dimensional model of the cluster structure, obtained by the Raymond-Smith code of the plasma emissivity; Sn90 use a $\beta$-model on the surface brightness profile. They quote a $\chi^{2}$ value of 287.2 (with 273 degree-of-freedom) and 20.1 (19), respectively. The latter method is also used by Sc92 on the ROSAT All Sky Survey data, and by JF84 and Br81 on the Einstein IPC data (cf. also fig. 8 in Sn90). In Sc92, both errors and $\chi^{2}$ values are not quoted. In JF84, the $\chi^{2}$ value is not indicated. Also in Br81, no $\chi^{2}$ value is indicated and the core radius is fixed to the one determined from the optical galaxy distribution [using a King model, Kent \& Sargent (1983) find a core radius of $11 \mathrm{arcmin}]$.

\begin{tabular}{lccc}
\hline Region & $\beta$ & $r_{\mathrm{c}}$ & ref \\
\hline$r<50^{\prime}$ & $0.73_{-0.09}^{+0.10}$ & $13.3_{-4.1^{\prime}}^{+4.8^{\prime}}$ & Cr97 \\
$10^{\prime}<r<78^{\prime}$ & 0.52 & $1.9^{\prime}$ & Sc92 \\
$8^{\prime}<r<50^{\prime}$ & $0.72_{-0.05}^{+0.08}$ & $12.5_{-2.8^{\prime}}^{+3.1^{\prime}}$ & Sn90 \\
$3.2^{\prime}<r<32^{\prime}$ & $0.57 \pm 0.03$ & $8.9^{\prime} \pm 1.7^{\prime}$ & $\mathrm{JF} 84$ \\
$10^{\prime}<r<17^{\prime}$ & $0.59 \pm 0.02$ & $8^{\prime}$ (fix) & Br81 \\
\hline
\end{tabular}

from optical velocity dispersion $\sigma_{\text {opt }}$ and X-ray spectral temperature $k T$ as

$\beta_{\mathrm{spec}}=\frac{\mu m_{\mathrm{p}} \sigma_{\mathrm{opt}}^{2}}{k T}$,

and, for an isothermal spherically-symmetric X-ray emission, from the surface brightness profile in the functional form (when $r \gg r_{\mathrm{c}}$ )

$S(r)=S_{0}\left[1+\left(\frac{r}{r_{\mathrm{c}}}\right)^{2}\right]^{0.5-3 \beta_{\mathrm{imag}}}$.

Edge \& Stewart (1991) calculated from the EXOSAT data on the Perseus cluster a $\beta_{\text {spec }}$ of $1.78_{-0.34}^{+0.48}$. Using the more recent reference values (with corresponding 1- $\sigma$ errors) on $\sigma_{\text {opt }}$ of $1026_{-64}^{+106} \mathrm{~km} \mathrm{~s}^{-1}$ (Fadda et al. 1996) and $k T=6.33_{-0.11}^{+0.13} \mathrm{keV}$ (Allen et al. 1992), we obtain $\beta_{\text {spec }}=1.09_{-0.14}^{+0.23}$. The significant decrease comes mainly from a much more careful determination of $\sigma_{\mathrm{opt}}$, once substructures, gaps in galaxy velocity distribution and more robust statistical tools are considered.

However, this estimate is much larger, for example, than $\beta_{\text {imag }}$ of about 0.52 , obtained from the azimuthally averaged profile of the cluster surface emission (Schwarz et al. 1992; but also cf. Table 4). The disagreement between $\beta_{\text {spec }}$ and $\beta_{\text {imag }}$ values is the reason for the so-called $\beta$ problem in the Perseus cluster (cf. also Allen et al. 1992). In particular, the non-symmetric emission from the cluster (as first pointed out by Branduardi-Raymont et al. 1981) traces a recent merger, that, together with the excess of gas to the East and of galaxies to the West, seems to emphasize the dependence of the $\beta$-problem on the sector analysed. In fact, Schwarz et al. (1992) observed a value of $\beta_{\text {imag }} \sim 0.5$ to West and North, and of $1.27_{-0.18}^{+0.29}$ to East (in their paper, there is no mention of the $\beta$ value to South).

We re-examine this issue, using our offset profiles out to about 80 arcmin from the X-ray peak. They are binned 


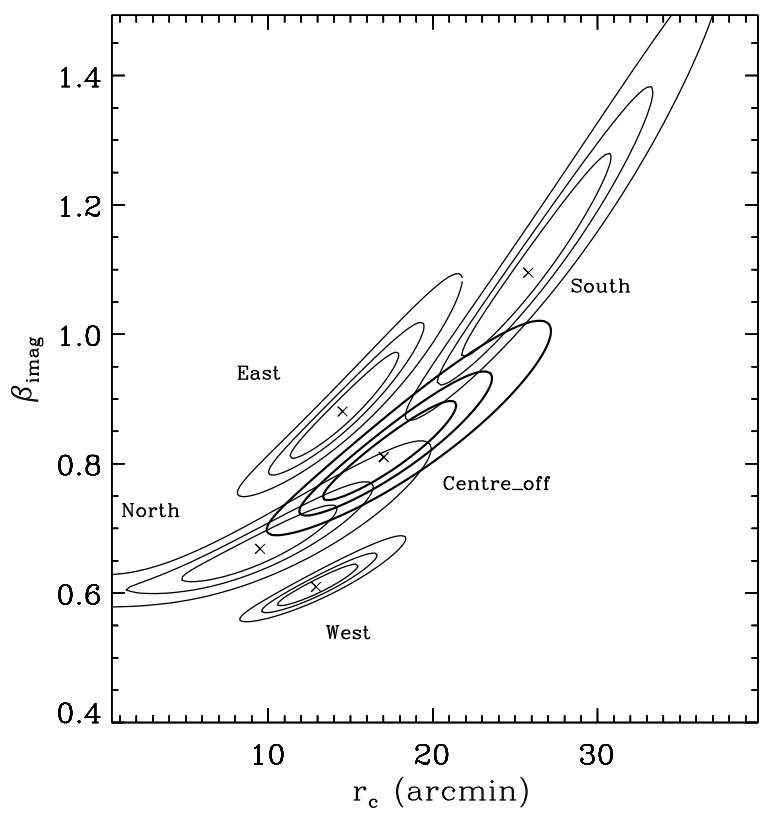

Figure 19. The 68, 90 and 99 per cent confidence contours in the $r_{\mathrm{c}}-\beta$ plane for the $\beta$-model applied on the $0.5-2 \mathrm{keV}$ surface brightness profile selected between 10 and 80 arcmin (cf. the last point on the thickest solid line in Fig. 18).

in width of [ 4, 3, 2, 1, (all the remaining at) 1] arcmin, due to the PSF behaviour in the outer part of PSPC.

First, an attempt is made to fit the profiles over the whole range of $[0,80]$ arcmin (cf. Table 5). Here, we note that, applying a $\beta$-model, the goodness-of-fit is generally very poor, in a $\chi^{2}$ sense. This is due to the fact that the statistical Poissonian errors related to the total counts present in each radial bin are particularly small (cf. Fig. 5). They do not consider any fluctuations present in each bin due to azimuthal variations within each sector. The contribution from these azimuthal fluctuations will be discussed in the next section.

Furthermore, as just noted in previous work (cf. Table 4), there is enhanced emission above the central part in all models. Thus, we have also analysed the surface brightness profiles avoiding the central 10 arcmin affected by the presence of the large cooling flow (as shown in the deprojection analysis, it has a typical $r_{\mathrm{cool}} \sim 0.2 \mathrm{Mpc}$, equivalent to about 6.5 arcmin; cf. also the colour ratios). As indicated in Table 5 , the reduced $\chi^{2}$ value decreases significantly but still remains too large to be statistically acceptable.

\subsubsection{Estimated error in the surface brightness mean value}

Previously, we have measured the error on the surface brightness as the Poissonian error related to the total counts in each radial bin. This is a statistical error that does not consider any fluctuation on the mean value (i.e., the surface brightness value) due to azimuthal variations intrinsic to the cluster.

The contribution expected from these fluctuations is particularly high in a non-relaxed cluster such as Perseus.

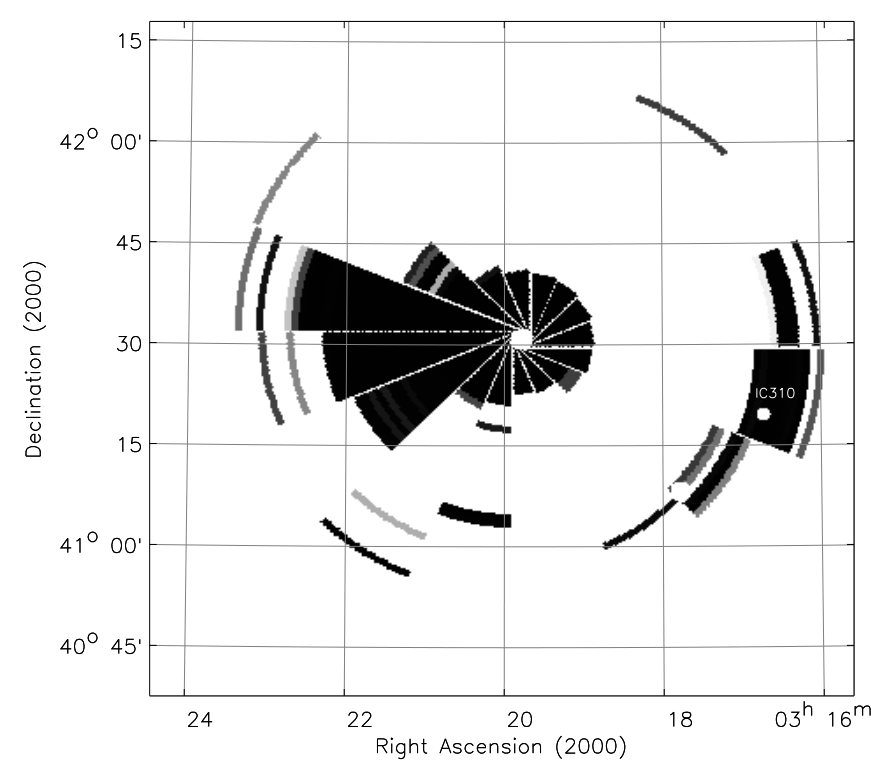

Figure 20. This is a $45^{\prime}$-radius map of the residuals significant at the 95 per cent confidence limit in terms of the $\chi^{2}$ probability distribution as drawn by equation (5). It is obtained after the mosaic of the four exposure-corrected image has been subtracted from the cluster emission simulated by a $\beta$-model with parameters $r_{\mathrm{c}}=17.0^{\prime}$ (68 per cent uncertainties range: 13.5, 21.3), $\beta=0.81(0.75,0.90)$, and $S_{0}=2.31 \times 10^{-2}$ counts s$^{-1}$ arcmin $^{-2}$. With a reduced $\chi^{2}$ of 0.05 , these values provide the best-fit to the Centre-off surface brightness profile, between 10 and 80 arcmin (cf. Table 5).

We have estimated the contribution from the dispersion of the mean values (assumed normally distributed) of the surface brightness calculated in $N_{\text {sect }}$ azimuthal sectors. This is done in each off-axis image (where the maximum angular size is about $90^{\circ}$ at the maximum width). Choosing $N_{\text {sect }}=4$ (i.e. angular sector of about $15^{\circ}$ ), we obtain a relative error of about 15 per cent over the radial range $[0,80]$ arcmin, i.e. about 10 times larger than the simple Poissonian error. The surface brightness profiles with the azimuthal fluctuations added in quadrature to the Poissonian errors are shown in Fig. 17.

After fitting these surface brightness profiles, we obtain values of reduced $\chi^{2}$ which are low due to the large, but real, errors now present in the data (cf. Tab. 4).

Of course, the value of $\chi_{\nu}^{2}$ is reduced by increasing $N_{\text {sect }}$, as fluctuations further dominate. On the other hand, it approaches a more statistically meaningful value of about 1 when both the angular region considered has width $\lesssim 15^{\circ}$ and only Poissonian error is considered. Then, the contribution from fluctuations is still larger by a factor of 3 and more.

In the following analysis, we quote the best-fit results obtained over each $90^{\circ}$-offset region (cf. Tab. 4), that can be considered as representative of the variations along different directions.

In Fig. 18, we plot the measured $\beta_{\text {imag }}$ and core radius for each sector, at different outer radii and for each of the 
Table 5. Best fit values of the surface brightness profiles and the corresponding reduced $\chi^{2}, \chi_{\nu}^{2}$, adopting different models. The values quoted are (scale radius -in arcminutes-, slope). Particularly, we present $\left(r_{\mathrm{c}}, \beta\right)$ for the $\beta$-model $(\beta) ;\left(r_{\mathrm{cut}} ; r_{\mathrm{P}}, \gamma ; r_{\mathrm{c}}, \beta\right)$ for the 'power law $+\beta^{\prime}$ model (pow $\left.+\beta\right) ;\left(r_{\text {cut }} ; r_{\mathrm{P} 1}, \gamma_{1} ; r_{\mathrm{P} 2}, \gamma_{2}\right)$ for a two broken power laws $\left(2\right.$ pow); $\left(r_{\mathrm{s}}, \eta\right)$ for the NFW gas density profile (NFW gas); $\left(r_{\mathrm{V}}, \alpha\right)$ for the de Vaucouleurs law (de Vauc).

\begin{tabular}{|c|c|c|c|}
\hline Region & model & values & $\chi_{\nu}^{2}$ \\
\hline \multicolumn{4}{|c|}{ only Poissonian error } \\
\hline North: $10^{\prime}<r<80^{\prime}$ & & $(8.8,0.65)$ & 1.90 \\
\hline $0^{\prime}<r<80^{\prime}$ & $\beta$ & $(3.6,0.58)$ & 6.67 \\
\hline West: $10^{\prime}<r<80^{\prime}$ & & $(12.5,0.60)$ & 12.87 \\
\hline $0^{\prime}<r<80^{\prime}$ & $\beta$ & $(2.0,0.51)$ & 51.15 \\
\hline South: $10^{\prime}<r<80^{\prime}$ & & $(25.3,1.05)$ & 2.60 \\
\hline $0^{\prime}<r<80^{\prime}$ & $\beta$ & $(2.2,0.53)$ & 71.12 \\
\hline East: $10^{\prime}<r<80^{\prime}$ & & $(24.8,1.23)$ & 8.55 \\
\hline $0^{\prime}<r<80^{\prime}$ & $\beta$ & $(4.4,0.60)$ & 70.89 \\
\hline Centre-off: $10^{\prime}<r<80^{\prime}$ & & $(17.5,0.85)$ & 15.42 \\
\hline $0^{\prime}<r<80^{\prime}$ & $\beta$ & $(3.4,0.57)$ & 122.38 \\
\hline \multicolumn{4}{|c|}{ Poissonian error and azimuthal fluctuations } \\
\hline North: $10^{\prime}<r<80^{\prime}$ & $\beta$ & $(9.5,0.67)$ & 0.12 \\
\hline \multirow[t]{5}{*}{$0^{\prime}<r<80^{\prime}$} & $\beta$ & $(4.3,0.62)$ & 0.17 \\
\hline & pow $+\beta$ & $(10.0 ; 3.5,1.21 ; 9.5,0.67)$ & 0.14 \\
\hline & 2 pow & $(15.0 ; 2.0,1.28 ; 4.3,1.87)$ & 0.18 \\
\hline & NFW gas & $(15.6,8.87)$ & 0.22 \\
\hline & de Vauc & $(0.017,0.29)$ & 0.13 \\
\hline West: $10^{\prime}<r<80^{\prime}$ & $\beta$ & $(12.9,0.61)$ & 0.37 \\
\hline \multirow[t]{5}{*}{$0^{\prime}<r<80^{\prime}$} & $\beta$ & $(2.7,0.52)$ & 1.00 \\
\hline & pow $+\beta$ & $(27.0 ; 3.3,1.38 ; 67.6,1.67)$ & 0.11 \\
\hline & 2 pow & $(26.0 ; 2.0,1.38 ; 4.3,1.77)$ & 0.34 \\
\hline & NFW gas & $(25.3,7.84)$ & 1.08 \\
\hline & de Vauc & $(0.001,0.23)$ & 0.54 \\
\hline \multirow{6}{*}{ South: $\begin{aligned} 10^{\prime} & <r<80^{\prime} \\
0^{\prime} & <r<80^{\prime}\end{aligned}$} & $\beta$ & $(25.8,1.10)$ & 0.19 \\
\hline & $\beta$ & $(21.7,0.99)$ & 0.72 \\
\hline & pow $+\beta$ & $(12.0 ; 3.4,1.39 ; 25.6,1.07)$ & 0.23 \\
\hline & 2 pow & $(26.0 ; 2.0,1.33 ; 6.1,2.39)$ & 0.32 \\
\hline & NFW gas & $(60.7,12.79)$ & 0.66 \\
\hline & de Vauc & $(3.33,0.67)$ & 0.54 \\
\hline \multirow{6}{*}{ East: $\begin{aligned} 10^{\prime} & <r<80^{\prime} \\
0^{\prime} & <r<80^{\prime}\end{aligned}$} & $\beta$ & $(14.5,0.88)$ & 0.40 \\
\hline & $\beta$ & $(10.1,0.79)$ & 0.64 \\
\hline & pow $+\beta$ & $(18.0 ; 3.5,1.22 ; 6.4,0.79)$ & 0.33 \\
\hline & 2 pow & $(19.0 ; 2.0,1.22 ; 5.7,2.30)$ & 0.33 \\
\hline & NFW gas & $(28.5,10.82)$ & 0.52 \\
\hline & de Vauc & $(0.42,0.46)$ & 0.50 \\
\hline \multirow{6}{*}{ Centre-off: $\begin{aligned} 10^{\prime} & <r<80^{\prime} \\
0^{\prime} & <r<80^{\prime}\end{aligned}$} & $\beta$ & $(\mathbf{1 7 . 0 , 0 . 8 1 )}$ & 0.05 \\
\hline & $\beta$ & $(4.7,0.63)$ & 0.54 \\
\hline & pow $+\beta$ & $(10.0 ; 3.4,1.24 ; 17.0,0.81)$ & 0.09 \\
\hline & 2 pow & $(22.0 ; 2.0,1.31 ; 5.2,2.12)$ & 0.14 \\
\hline & NFW gas & $(20.3,9.37)$ & 0.58 \\
\hline & de Vauc & $(0.045,0.33)$ & 0.13 \\
\hline
\end{tabular}

three energy bands. Thus, we check also the dependence of $\beta_{\text {imag }}$ upon the colours, that we expect if any merging action is produced by substructure with a significant difference in temperature from the ICM.

Using the $\beta$-model for the analysis of the different sectors, we find a value around 0.65 in the North, and an increase from the exterior to the centre, from 0.45 to 0.65 , in the West.

The eastern and southern regions provide values of about 1 over all radial ranges considered. These values, as shown by Edge \& Stewart (1991), indicate an on-going merger that seems to involve the eastern region (cf. again the conclusion in Schwarz et al. 1992) as well as the southern one. In particular, the soft profile steepens significantly around 40 arcmin to the East. The colour analysis shows a gradient that agrees with a softening of the emission (cf. Fig. 16.).

Due to the correlation in the $\beta-r_{\mathrm{c}}$ plane (cf. Fig. 18), the core radii emphasize a peculiar gas distribution in these 2 sectors: they are about twice the ones estimated in the North and West. The presence of this correlation can also partially explain the disagreement between the parameters fitted in the previous work, where the lower core radii correspond to lower $\beta$ (cf. Table 4).

Fig. 18 also shows that, although there is a trend for the gas distribution to be flatter in the medium band, the surface brightness selected in the energy range 0.9-1.3 keV shows a steeper profile in the East sector, underlining the 

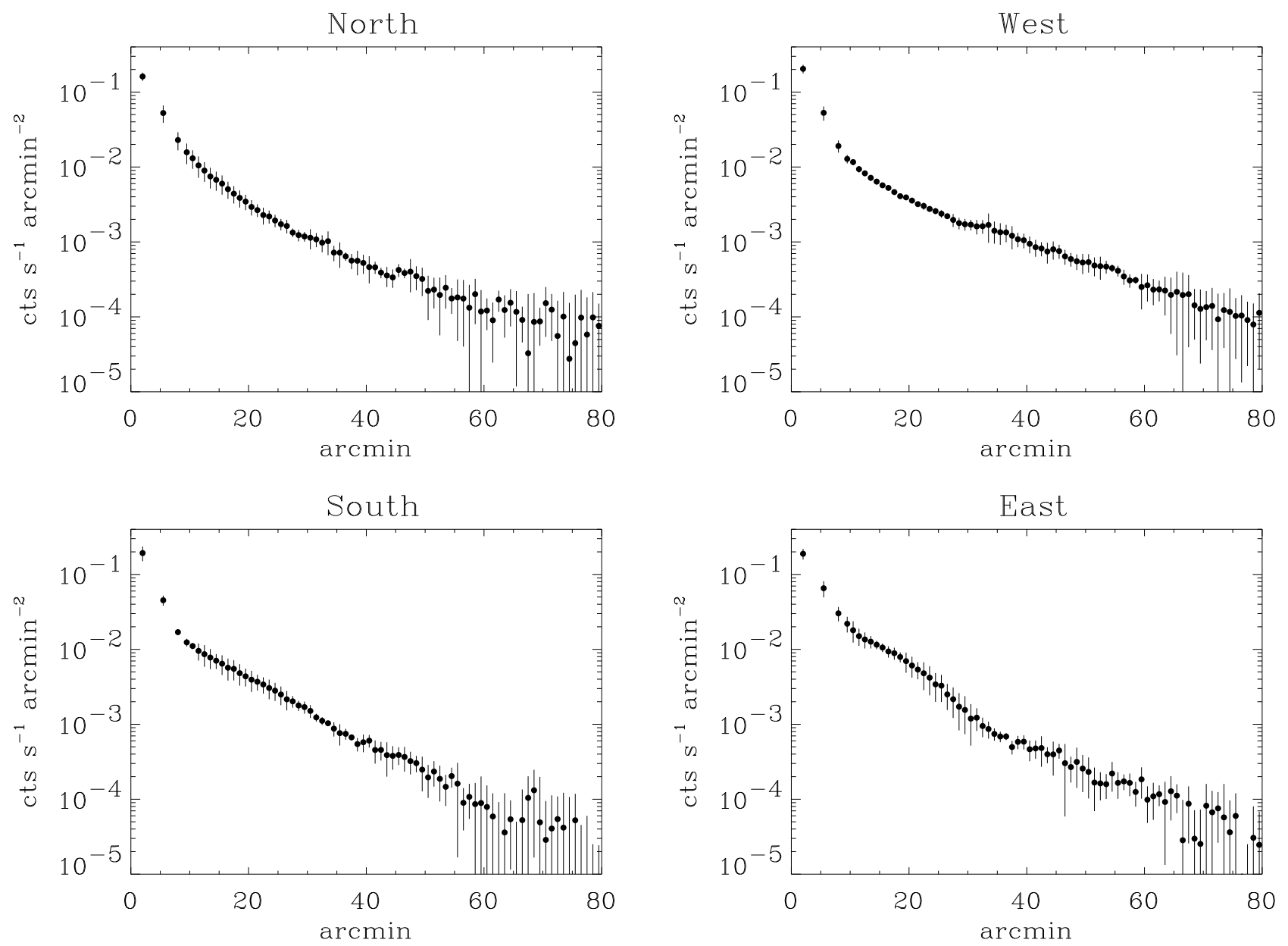

Figure 17. The [ 4, 3, 2, 1, (all the remaining) 1] arcmin plots with the errors obtained considering also the azimuthal fluctuations within each radial bin. These plots are fitted with the $\beta$-model and the other models discussed in section 2.4. The results of the fitting procedure are presented in Table 5.

ICM interaction with a clump of gas at temperature greater than $1 \mathrm{keV}$

The $0.5-2.0 \mathrm{keV}$ band indicates a $\beta_{\text {imag }}$ value consistent with the average of the 3 other values calculated in the different bands.

The errors on the two interesting parameters, $\beta_{\text {imag }}$ and $r_{\mathrm{c}}$, are shown in Fig. 19 as contours plotted at 68, 90 and 99 per cent of confidence level (i.e. $\chi^{2}$-value regions of $\chi_{\min }^{2}+$ $2.30, \chi_{\min }^{2}+4.61$ and $\chi_{\min }^{2}+9.21$, respectively; e.g. Press et al. 1992, page 687). They are estimated with the fit performed on the surface brightness profile selected between 10 and 80 arcmin in $0.5-2.0 \mathrm{keV}$ band. No agreement is evident, at the 99 per cent confidence level, among the different sectors considered. Thus, the complexity of the area in terms of the gas distribution is once more emphasized. However, a good representation of the region is provided through the azimuthally averaged Centre-off profile.

With these new constraints on the $\beta_{\text {imag values in the }}$ different sectors of A426, we can address the issue of the $\beta$-problem.

\subsubsection{Resolution of the $\beta$ problem}

Following Bahcall \& Lubin (1994), and the recent results on N-body simulations of clusters of galaxies (cf. Thomas et al. $1998)$, we first consider a correction to the $\beta_{\text {imag value. The }}$ $\beta$-model, as described by equation (3), has been introduced assuming that the gas and the galaxies are in hydrostatic equilibrium with the cluster potential, and that the galaxy distribution is well described by the King approximation (1962) to the isothermal sphere. This approximation is proportional to $r^{-3}$ at the outer radii, and does not match the dark matter dependence of $r^{-2.4}$, which is determined from N-body simulations (cf. Fig. 8). Then, the hypothesis that the optical light traces the mass, i.e. that the galaxy number density profile describes the gravitational profile of the cluster over the virialized region (as the recent CNOC results also seem to confirm; cf. Carlberg et al. 1997), indicates a way to correct the $\beta_{\text {imag }}$ value to give the correct dependence on the galaxy distribution of the radius:

$$
\begin{aligned}
& \rho_{\text {gas }}(r)=\rho_{\text {gal }}(r)^{\beta_{\text {imag }}} \propto r^{-3 \beta_{\text {imag }}} \propto r^{-2.4 \beta_{\text {imag,corr }}}, \\
& \text { i.e., } \beta_{\text {imag,corr }}=1.25 \beta_{\text {imag }} .
\end{aligned}
$$



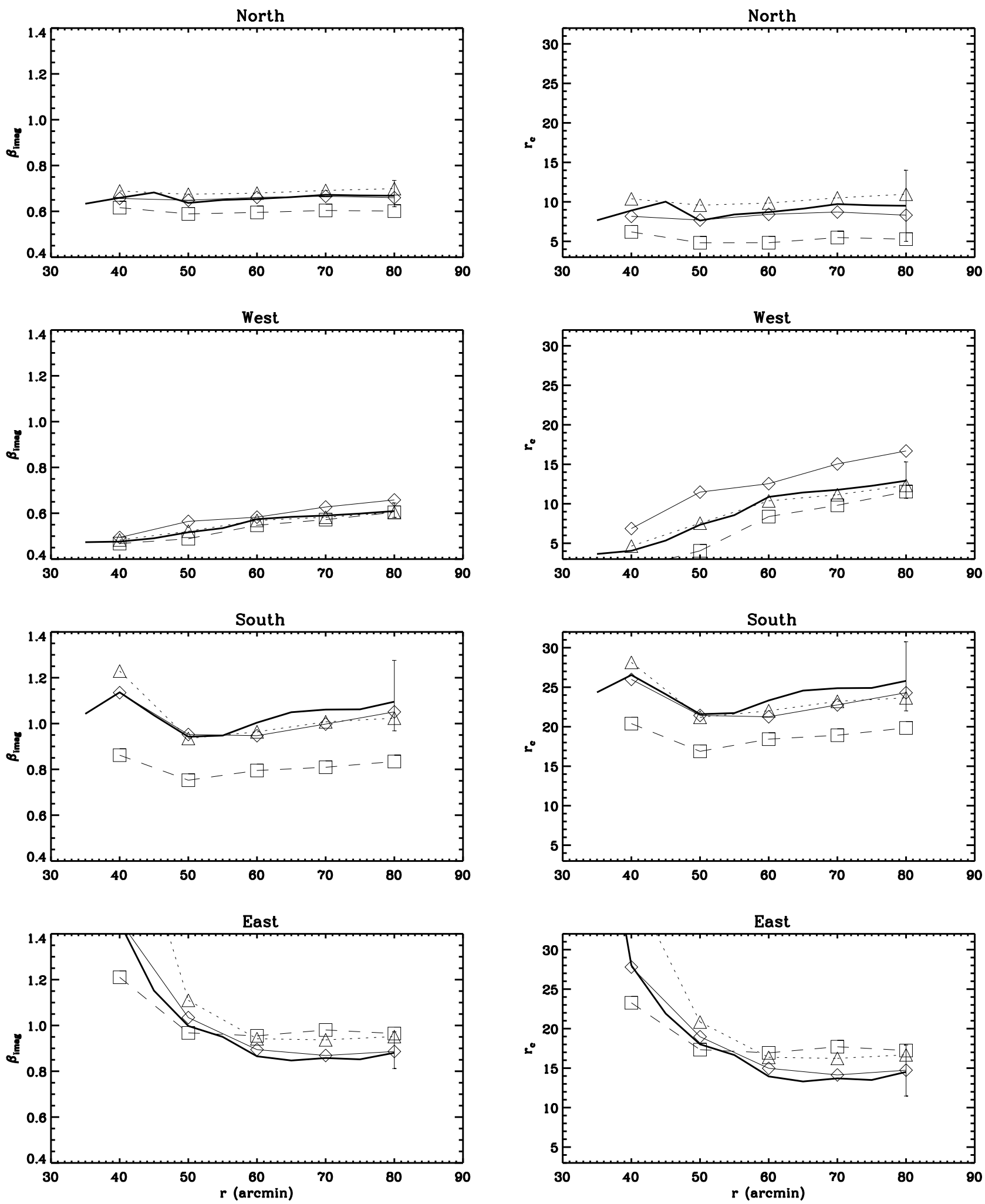

Figure 18. These plots show the results on the $\beta$ value and the core radius, $r_{\mathrm{c}}$, when the $\beta$-model is fitted on the profiles binned in [ $4,3,2,1$, (all the remaining) 1] arcmin and selected between 10 arcmin and different outer radius in the 3 energy bands: soft (triangles joined by a dotted line); medium (dashed line and squares); hard (solid line and diamonds). The thickest solid line represents the result when the $\beta$-model is applied to the $0.5-2.0 \mathrm{keV}$ exposure-corrected image. At the last point on this line are related the error-bars drawn. These plots show a correlation between $\beta$ and $r_{\mathrm{c}}$ (see also Jones \& Forman 1984). 
Thus, we have compared $\beta_{\text {spec }}=1.09_{-0.14}^{+0.23}$ with the corrected values of $\beta_{\text {imag }} ; 0.84,0.76,1.37,1.10,1.01$ (with 68 per cent errors of about 10 per cent) for the North, West, South, East and Centre-off, respectively. There is now a reasonable agreement between $\beta_{\text {imag,corr }}$ and $\beta_{\text {spec }}$, which eliminates the $\beta$ problem in the Perseus cluster.

Furthermore, assuming the equation $\beta_{\text {spec }}=\beta_{\text {imag,corr }}$ is valid, we can calculate a velocity dispersion, $\sigma_{\text {imag, }}$, us-

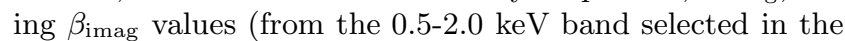
radial range 10-80 arcmin) and an isothermal temperature of $6.3 \mathrm{keV}$. These values are quoted in square brackets in Table 3, and show a good agreement with the deprojection estimates, $\sigma_{\mathrm{dpr}}$. All the velocity dispersions calculated so, are also marginally consistent with the optically determined velocity dispersion, $\sigma_{\text {opt }}$, of $1026_{-64}^{+106} \mathrm{~km} \mathrm{~s}^{-1}$.

Using the best-fit results from the $\beta$-model, we investigate in Fig. 20 the residuals (significant at the 95 per cent confidence level) for a cluster simulated by using a $\beta$ - model, $S(x, y)$, compared to the raw data, $I(x, y)$. Both $S(x, y)$ and $I(x, y)$ are in counts pixel ${ }^{-1}$. Then, the significance is assessed in terms of a $\chi^{2}$ distribution:

$\chi^{2}=\sum_{j=1}^{N_{x} \times N_{y}} \frac{\left[I\left(x_{j}, y_{j}\right)-S\left(x_{j}, y_{j}\right)\right]^{2}}{S\left(x_{j}, y_{j}\right)}$,

where $N_{x}$ and $N_{y}$ are the numbers of pixels in the rows and columns in the selected sectors of $22^{\circ} .5$ in annular circular bins of $[4,3,2,1$, (all the remaining) 1] arcmin.

From this map, apart from the central cooling region which contributes to the total luminosity $L_{\text {bol }}=2.8 \times 10^{45}$ erg $\mathrm{s}^{-1}$ about 50 per cent (cf. the deprojection results in in Fig. 9), the eastern excess extends to 40 arcmin from the centre with an estimated luminosity in the residuals of about $8 \times 10^{43} \mathrm{erg} \mathrm{s}^{-1}$. From the $L-T$ relationship in the groups and clusters sample of Mulchaey and Zabludoff (1998), this luminosity corresponds to about $2 \mathrm{keV}$, as we also conclude from the colour analysis. Other extended emission appears related to the galaxy IC310, at about 40 arcmin westwards (IC310 is the masked point source; see also Cruddace et al. 1997). This clump has an estimated luminosity of $\sim 1 \times 10^{43}$ erg s $\mathrm{s}^{-1}$ with a corresponding temperature of $1 \mathrm{keV}$. This is further support for the drastic increase in the colour ratio values for the same region is attributed to an excess of cool gas, as seen in Fig. 14. Both of these clumps appear as groups still merging with the cluster.

Other poorly defined discrepancies with an azimuthally averaged $\beta$-model profile appear in the South. They can be part of the eastern excess and are responsible of the $\beta$ value of about unity measured in that sector.

We stress that these residuals depends on the assumption of the model. As shown in Section 2, the residuals are not significant if the elliptical isophotes are allowed to have free centroids. Furthermore, modelling this elliptical isophotal intensity profile with a $\beta$-model in the range [10-80] arcmin, we obtain $\beta=0.63 \pm 0.01$ and $r_{\mathrm{c}}=9.3 \pm 0.2$ arcmin, for a reduced $\chi^{2}$ of 33.6. These values agree well with those observed for the sector to the North, that appears as the most relaxed region in the cluster.

\subsection{On the fitting of the surface brightness profile}

The inadequacy of the $\beta$-model in reproducing the gas profile over the whole radial range, in particular in the central part, has driven us to look for other possible functional forms.

We consider the following models:

(i) a power law plus a $\beta$ model, to describe the inner cooling region and the outer part, respectively, with a radius of cut-off, $r_{\text {cut }}$, as a free parameter:

$S_{\mathrm{b}}= \begin{cases}S_{0}\left(\frac{r}{r_{\mathrm{P}}}\right)^{1-2 \gamma} & \text { if } r<r_{\mathrm{cut}} \\ S_{0}\left[1+\left(\frac{r}{r_{\mathrm{c}}}\right)^{2}\right]^{0.5-3 \beta} & \text { if } r>r_{\mathrm{cut}}\end{cases}$

where the exponent for the 'power law' model comes from the consideration that

$\rho_{\text {gas }}=\rho_{0}\left(\frac{r}{r_{\mathrm{P}}}\right)^{-\gamma} \longrightarrow S_{\mathrm{b}}=S_{0}\left(\frac{r}{r_{\mathrm{P}}}\right)^{1-2 \gamma}$;

(ii) two density power laws, boken at radius $r_{\text {cut }}$;

(iii) a generalized form of the de Vaucouleur's law (1948):

$S_{\mathrm{b}}=S_{0} \exp \left[-\left(\frac{r}{r_{\mathrm{V}}}\right)^{\alpha}\right]$.

We also introduce a gas density profile obtained from the NFW dark matter profile when the hydrostatic equation under the isothermal condition is applied:

$\rho_{\text {gas }}=\rho_{0}(1+x)^{\eta / x}$,

where $x=r / r_{\mathrm{s}}$ and $\eta=4 \pi G \rho_{\mathrm{s}} r_{\mathrm{s}}^{2} \mu m_{\mathrm{p}} /(k T)$ (cf. Makino, Sasaki \& Suto, 1998, and Ettori \& Fabian, 1998). The surface brightness profile is then obtained by numerical integration of the gas density profile in equation (9).

We use the reduced $\chi^{2}$ provided by each model to assess their suitability. The best-fit parameters are listed in Table 5. From this table, the 'power law $+\beta$ model' appears as the more appropriate description of the data over all the range of radii. In terms of the $F$-test, when we compare the $\beta$ model with the 'power law $+\beta$ ' fit, we obtain that it is still a good description of the northern data, but it becomes worst at 99 per cent of confidence level in the cases of the West, South, East and Centre-off. Once again (cf. the colour analysis and Fig. 18), the North appears as the more relaxed region in the Perseus cluster.

We also note that the 'power law $+\beta$ model' highlights the merger of the clump of gas around IC310 in the outer region to West. In fact, the $\beta$ value increases there to about 1.4, compared to a value of 0.6 from the single $\beta$ model. Moreover, the cut-off radius is 26 arcmin to West and 18 arcmin to East (around 12 in the other sectors), underlining the elliptical configuration discussed in Section 2.

However, the $\beta$ model is a poor description when it is also compared to the models (ii) and (iii), but it is better than the gas profile obtained from the NFW law. In fact, this latter profile shows the greatest disagreement with the data. We check elsewhere on the consistency of the gas distribution obtained from NFW dark matter potential with a larger set of X-ray cluster profiles.

The slope in $\rho_{\text {gas }} \propto r^{-\alpha}$ is in the range $[1.22,1.38]$ in the core region and become steeper to $[1.77,2.39]$ in the outer part of the Perseus cluster. There, the slopes from the two broken power laws are consistent, within an average error of 15 per cent, with the asymptotic behaviour of the $\beta$ model 


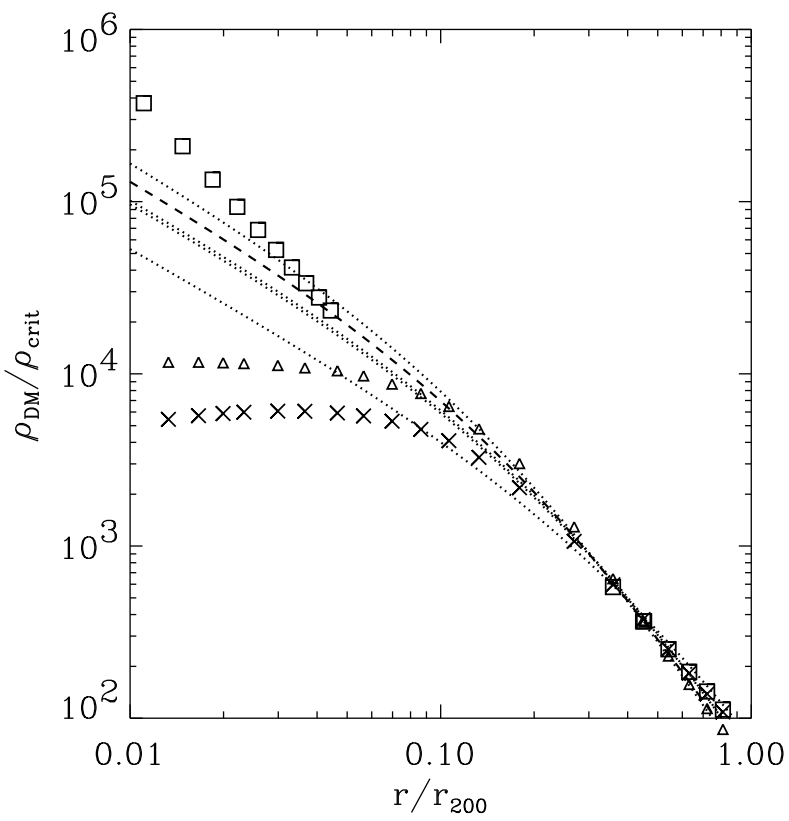

Figure 22. The dark matter density profiles as obtained from the best-fit values of the NFW gas profile are the dashed line (Centre-off) and the dotted lines (the other four sectors). The crosses represent the result from the $\beta$ model, the triangles are from the deprojection analysis, and the squares what we obtain applying two broken power laws (we plot just the external values for a more clarity). All of them are obtained in the case of Centreoff.

(apart from the southern region where the discrepancy is 28 per cent).

\section{THE CONSTRAINTS ON THE MASS PROFILE}

The form of the gravitational potential remains the most uncertain element of the deprojection analysis, since the observational constraints are not completely sufficient. However, some conclusions on the shape of the dark matter profile can be achieved under the assumptions of hydrostatic equilibrium and isothermality of the gas distribution in the cluster. Then, the total gravitating mass, responsible for the external potential that maintains the gas mass within a radius $R$ in equilibrium, is written as

$M_{\mathrm{tot}}=\int_{0}^{r} 4 \pi \rho_{\mathrm{DM}} r^{2} d r=-\frac{r^{2}}{G \rho_{\mathrm{gas}}} \frac{d P_{\mathrm{gas}}}{d r}$.

This equation, together with the perfect gas law, is used in Fig. 21 to describe how the total gravitating mass depends on the radius, applying both the deprojection technique and the $\beta$-model. The results indicate consistency between the total mass, estimated by these methods, and previous estimates (when the errors are properly considered). In the same figure, the mass distribution, as obtained through the deprojection analysis, emphasizes the over-density in the east sector where the merging group acts.

In Fig. 22, we plot the dark matter density profile as obtained from (i) the best-fit $\left(\eta, r_{\mathrm{s}}\right)$ values of NFW gas profile, once the definition of $\eta$ is used with a plasma temperature of $6.3 \mathrm{keV}$; (ii) the best-fit slopes $\gamma$ from the two broken power laws model, given that $M_{\text {tot }} \propto \gamma k T r$ and $\rho_{\mathrm{DM}} \propto \gamma k T r^{-2}$; (iii) the $\beta$-model, in which $M_{\text {tot }} \propto \beta k T r^{3} /\left(r^{2}+r_{\mathrm{c}}^{2}\right)$ and $\rho_{\mathrm{DM}} \propto \beta k T\left(r^{2}+3 r_{\mathrm{c}}^{2}\right) /\left(r^{2}+r_{\mathrm{c}}^{2}\right)^{2}$; (iv) the result of the deprojection analysis using the potential from the true isothermal sphere. Here we note that both the $\beta$ and the power law models indicate a dark matter density profile that is flatter than N-body simulations profile, with a dependency upon radius of slope 2 , instead of about 2.4. However, on this radial range, this affects slightly the determination of the total mass as shown in Fig. 21.

From fitting the core with a power law, it appears more convenient to use a steeper profile than the NFW one, whereas the King profile provides a plateau (cf. also Fig. 8). Furthermore, the best-fit values define $r_{200}$, the radius at which the average over-density in the cluster is 200, equal to about $2.7 \mathrm{Mpc}$. This means that we are mapping, in our analysis, the region where the over-density is about 300 or more.

The results in Fig. 22 show good agreement within the region $0.4-0.8 r_{200}$, i.e. above the core region that depends upon the functional form of the potential adopted and within the radial limits of our observations.

These constraints on the total mass are applied to describe the distribution of the gas fraction, $f_{\text {gas }}$, shown in Fig. 23 , in dependence both of the central azimuthally averaged observations and of each sector considered. We obtain a gas contribution to the total mass of 30 per cent at 2.3 Mpc. This value is consistent with the Spartan 1 estimate at $1.5 \mathrm{Mpc}$ of about 24 per cent (from Fig. 3 in Cruddace et al. 1997).

Here we note that if any decrease in the average temperature of the cluster plasma is detected in the outskirts, as appears common in clusters from the recent analyses of $A S C A$ data by Markevitch et al. (1997), then the gas fraction obtained assuming an isothermal profile should be increased significantly due to the corresponding reduction of gravitating mass. However, from the colour analysis, we observe that the gas temperature in Perseus does not decrease 3 with respect to the reference value of $6.3 \mathrm{keV}$ up to 50 arcmin (about $10 r_{\mathrm{c}}$ ), at least in the North and South. In the other two sectors, we detect cool emission from extended and identified components, probably due to a merger that could affect the hydrostatic assumption.

The latter two excesses in emission are also seen in the plots of the gas fraction (Fig. 23), where the distribution of each sector is compared to the azimuthally averaged value. The eastern excess, between 0.5 and $1 \mathrm{Mpc}$, corresponds to the merging group, while the excess in the West, where the gas fraction reaches a value of about 40 per cent at 2.3 Mpc, shows a discrepancy with the azimuthally averaged value over all the radial range outside $1 \mathrm{Mpc}$. This is also shown in Fig. 20, where the contribution from the extended emission around IC310 becomes significant.

$\S$ using the same analysis, it is difficult to put any upper limit; cf. Fig. 11 

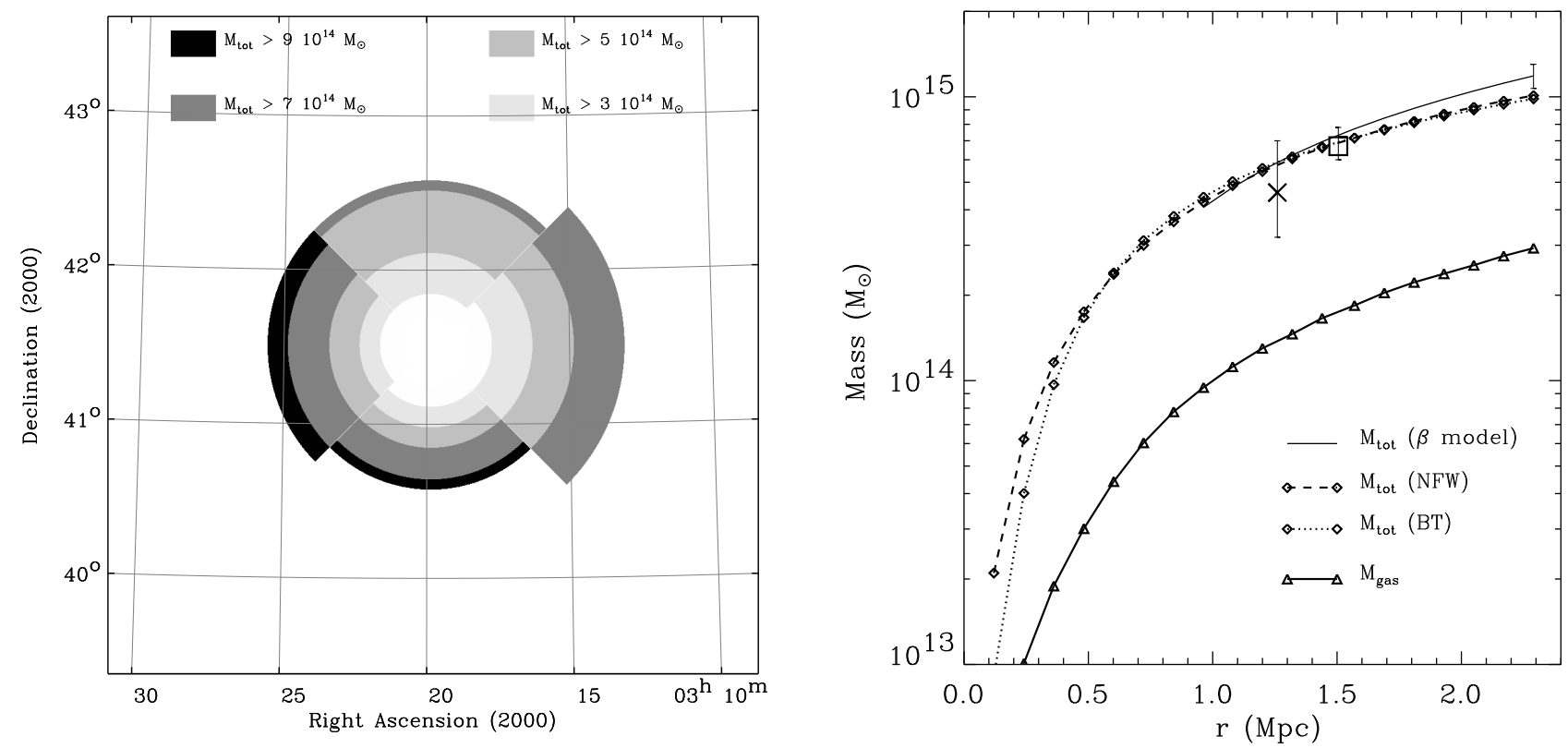

Figure 21. These plots present the mass distribution how has been obtained applying the deprojection technique, under the hydrostatic and isothermal assumptions. (left) The map is a combination of the four off-axis observations results on the total gravitating mass. (right) The mass profiles obtained by applying both the deprojection technique and the best-fit results from the $\beta$-model on the azimuthally averaged surface brightness profiles are here compared with the previous estimates: about $5 \times 10^{14} M_{\odot}$ at $\mathrm{R}=1.3 \mathrm{Mpc}(\mathrm{cross}$; Eyles et al. 1991); $6.7 \times 10^{14} M_{\odot}$ at $1.5 \mathrm{Mpc}$ (square; from Cruddace et al. 1997 after we adopt the redshift of 0.0178 ). The last point at $2.3 \mathrm{Mpc}$ reports a value of $1.19_{-0.12}^{+0.12} \times 10^{15} M_{\odot}$, for the range of the 10th and 90th percentile on 100 Monte-Carlo simulated estimations (see Sect. 4).

\section{CONCLUSION}

Our main conclusions are as follows:

(i) The distribution of the ICM in the Perseus cluster is complex, being more homogeneous in the northern region, with clear excess in the eastern part. We can not confirm any temperature higher than about $6 \mathrm{keV}$ in the North, or North-West areas, as claimed from the $A S C A$ results.

The analysis of the PSPC colour ratios indicates an isothermal profile around $6 \mathrm{keV}$ within 50 arcmin, a significant decrease in the cooling flow region, and fluctuations both in the eastern (between 20 and 50 arcmin from the $\mathrm{X}$-ray center) and in the western areas (around IC310, 3550 arcmin from the center), where we detect cool extended emission with luminosity of few times $10^{43} \mathrm{erg} \mathrm{s}^{-1}$. This result is also supported from a $\beta$-model analysis and shows the presence of groups still merging with the main body of the cluster. This merger event produces a disturbance of the gas within the gravitational potential, that can be modelled by elliptical isophotes with free centroids. This model then eliminates significant residuals from the regions of the clumps, emphasizing that these features must rather be entities within the cluster, and not foreground / background objects.

Furthermore, we show that the observed discrepancy in surface brightness can not be justified simply in the basis of the gradient in absorption present over the region, even through some observed gradient is present as detected from our colour analysis.

(ii) We use the $\beta$-model to highlight the disturbed intra- cluster gas distribution in the East, South-East. The bestfit parameter $\beta_{\text {imag }}$ is shown to be dependent on the outer radius in the extraction of the surface brightness profile, although it converges outside 50 arcmin $(1.5 \mathrm{Mpc})$. The disagreement between $\beta_{\text {spec }}$ and $\beta_{\text {imag }}$ disappears when $\beta_{\text {spec }}$ is properly evaluated with a recent estimate of the optical velocity dispersion that takes into account substructures in the velocity space, and $\beta_{\text {imag }}$ is carefully estimated in the outer part of the cluster with a proper correction for the slope of the dark matter density profile.

Thus, we can claim that the $\beta$-problem in the Perseus cluster is solved.

(iii) The surface brightness profile in the Perseus cluster does not seem to be well described by a single $\beta$-model over all the range $[0,80]$ arcmin. This is common for cooling flow clusters that also show merging in the outskirts, as in Perseus.

After testing several different models, we find that the best statistical agreement between the data and the gas distribution is obtained with a model with a power law fitted to the inner cooling region, and a $\beta$ model for the outer component.

We also apply of the gas profile obtained using the NFW dark matter potential in the hydrostatic equation. However, this does not provide an acceptable description of the Perseus data, due to the no-relaxed state of the cluster.

(iv) With the assumption of hydrostatic equilibrium, we place constraints on the non-luminous mass density profile from the best-fit results of the NFW gas profile, $\beta$-model, 

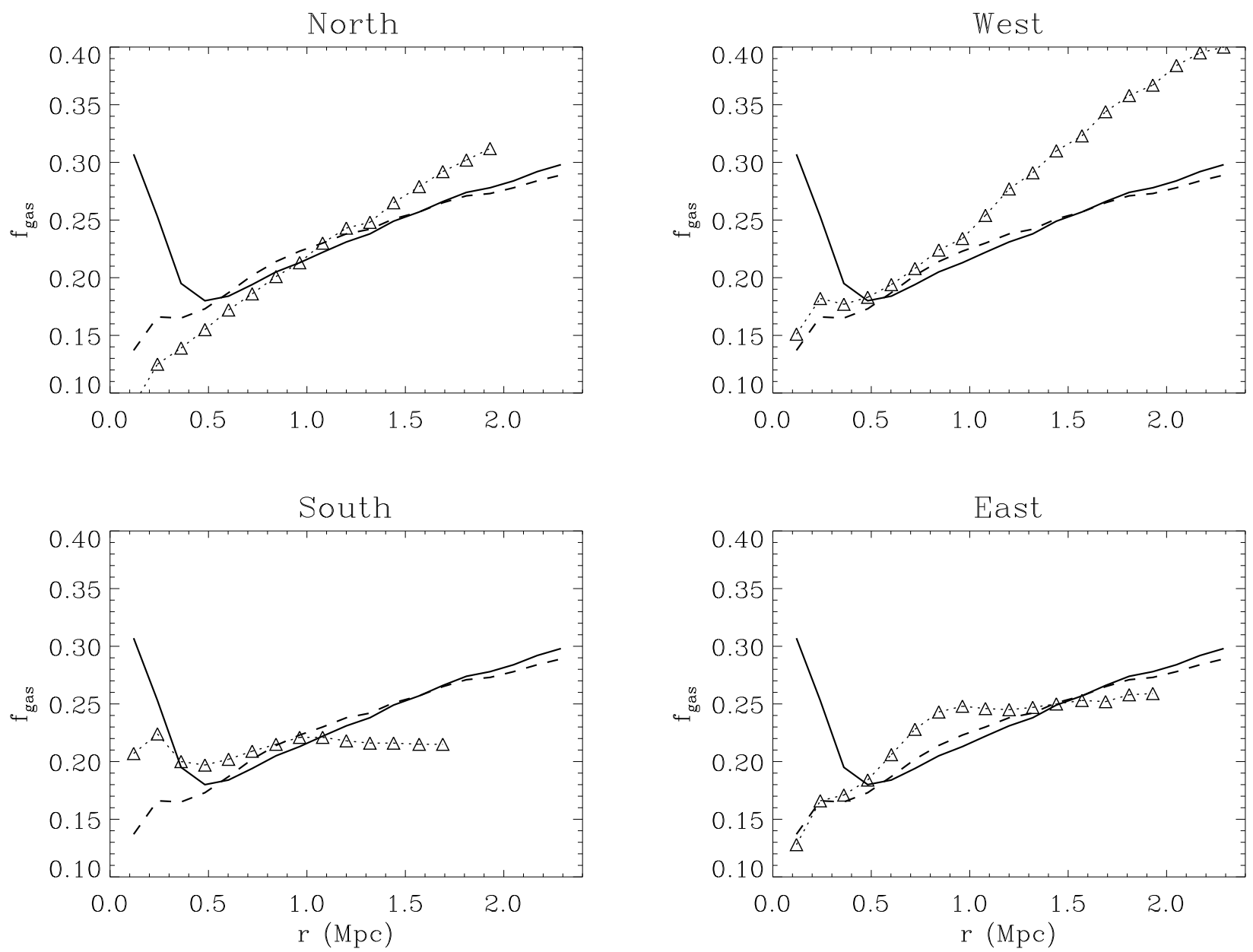

Figure 23. These plots show the gas fraction, $f_{\text {gas }}$, as function of the radius $R$ for the four sectors deprojected by assuming a NavarroFrenk-White profile dark matter density profile (triangles joined by a dotted line). We also show, as comparison, the value for the gas fraction measured in the azimuthally averaged deprojected image, both using a BT profile (solid line) and a NFW profile under isothermal assumption (dashed line). (Cf. also Table 3 for the values of $f_{\text {gas }}$ quoted at $R_{\text {out }}$ and the relative error). At $1.5 \mathrm{Mpc}$, Cruddace et al. (1997) calculate a value of about 0.24 .

two broken power laws model, and the conditions provided from the deprojection analysis.

All of these are consistent in defining $r_{200}$ at $2.7 \mathrm{Mpc}$.

The total mass in cluster within $2.3 \mathrm{Mpc}\left(0.85 r_{200}\right)$ is $1.19_{-0.12}^{+0.12} \times 10^{15} M_{\odot}$, where the errors are the 10 th and 90 th percentile calculated estimating 100 times the mass through the $\beta$-model, once the temperature is randomly selected around its best value and the errors are considered, the core radius is also randomly selected and $\beta$ is fixed from the best fit on the surface brightness profile. The different models agree (within the respective error) on this estimate, even if a slight difference $(\sim 20$ per cent $)$ is present in the slope of their dark matter profiles in the range of $0.4-0.8 r_{200}$, where our observations of the gas profile are available and not affected from the core region.

(v) Using constraints on the gas and non-luminous mass distribution, we measure the gas fraction to be equal to about 30 per cent at $2.3 \mathrm{Mpc}$. This is similar to that observed in Coma and other clusters (White et al. 1993, White \& Fabian 1995), and disagrees with the primordial nucleosynthesis estimates of the baryon fraction in the Universe (e.g. Olive 1996), unless the ratio between the total density and the critical value, $\Omega_{0 \text {,matter }}$, is less than 0.2 .

\section{ACKNOWLEDGEMENTS}

We are grateful to the members of the IoA X-ray Group for useful discussions. SE acknowledges support from PPARC and the Cambridge European Trust, ACF the support of the Royal Society and DAW that of PPARC. This research has made use of data obtained through the High Energy Astrophysics Science Archive Research Center Online Service, provided by the NASA-Goddard Space Flight Centre.

\section{REFERENCES}

Allen, S.W., Fabian, A.C., Johnstone, R.M., Nulsen, P.E.J. \& Edge, A.C., 1992, MNRAS, 254, 51

Allen, S.W. \& Fabian, A.C., 1994, MNRAS, 269, 409

Allen, S.W. \& Fabian, A.C., 1997, MNRAS, 286, 583

Andreon, S., 1994, A\&A, 284, 801 
Arnaud, K.A. et al., 1994, ApJ, 436, L67

Arnaud, K.A., 1996, Astronomical Data Analysis Software and Systems V, eds. Jacoby G. and Barnes J., p. 17, ASP Conf. Series vol. 101

Bahcall, N.A. \& Lubin, L.M., 1994, ApJ, 426, 513

Binney, J. \& Tremaine, S., 1987, Galactic Dynamics, Princeton University Press, p. $226 \mathrm{f}$

Branduardi-Raymont, G., Fabricant, D., Feigelson, E., Gorenstein, P., Grindlay, J., Soltan, A. \& Zamorani, G., 1981, ApJ, 248,55

Buote, D.A. \& Canizares, C.R., 1996, ApJ, 457, 565

Carlberg, R.G. et al., 1997, ApJL, 485, L13

Cavaliere, A. \& Fusco-Femiano, R., 1976, A\&A, 49, 137

Cruddace, R.G., Kowalski, M.P., Fritz, G.G., Snyder, W.A., Fenimore, E.E. \& Ulmer, M.P., 1997, ApJ, 476, 479

de Vaucouleurs, 1948, Ann. Astrophys., 11, 247

Dickey, J.M. \& Lockman, F.J., 1990, ARAA, 28, 215

Edge, A.C. \& Stewart, G.C., 1991, MNRAS, 252, 428

Ettori, S. \& Fabian, A.C., 1998, MNRAS, submitted

Eyles, C.J., Watt, M.P., Bertram, D., Church, M.J., Ponman, T.J., Skinner, G.K. \& Willmore, A.P., 1991, ApJ, 376, 23

Fabian, A.C., Hu, E.M., Cowie, L.L. \& Grindlay, J., 1981, ApJ, 248,47

Fadda, D., Girardi, M., Giuricin, G., Mardirossian, F. \& Mezzetti, M., 1996, ApJ, 473, 670

Forman, W., Kellogg, E., Gursky, H., Tananbaum, H. \& Giacconi, R., 1972, ApJ, 178, 309

Gursky, H., Kellogg, E., Leong, C., Tananbaum, H. \& Giacconi, R., 1971, ApJL, 165, L43

Hasinger, G., Boese, G., Predehl, P., Turner, T.J., Yusaf, R., George, I.M. \& Rohrbach, G., 1993, MPE/OGIP Calibration Memo CAL/ROS/93-015

Jones, C. \& Forman, W., 1984, ApJ, 276, 38

Kaastra, J.S., 1992, An X-Ray Spectral Code for Optically Thin Plasmas (Internal SRON-Leiden Report, updated version 2.0)

Kent, S.M. \& Sargent, W.L.W., 1983, AJ, 88, 697

King, I. R., 1962, AJ, 67, 471

Kowalski, M.P., Cruddace, R.G., Snyder, W.A. \& Ulmer, M.P., 1993, ApJ, 412, 489

Liedahl, D.A., Osterheld, A.L. \& Goldstein, W.H., 1995, ApJ, 438, L115

Makino, N., Sasaki, S. \& Suto, Y., 1998, ApJ, 497, 555

Malumuth, E. M. \& Kirshner, R.P., 1985, ApJ, 291, 8

Markevitch, M., Forman, W.R. Sarazin. C.L. \& Vikhlinin, A., 1997, ApJ, submitted (astro-ph/9711289)

Mohr, J.J., Fabricant, D.G. \& Geller, M.J., 1993, ApJ, 413, 492

Morrison, R. \& McCammon, D.M., 1983, ApJ, 270, 119

Mulchaey, J.S. \& Zabludoff, A.I., 1998, ApJ, 496, 73

Navarro, J.F., Frenk, C.S. \& White, S.D.M., 1995, MNRAS, 275, 720

Olive, K.A., 1996, astro-ph/9609071

Peres, C.B., Fabian, A.C., Edge, A.C., Allen, S.W., Johnstone, R.M. \& White, D.A., 1998, MNRAS, in press

Press, W.H., Teukolsky, S.A., Vetterling, W.T. \& Flannery, B.P., 1992, Numerical Recipes, Cambridge University Press

Primini, F., et al., 1981, ApJL, 243, L13

Raymond, J.C. \& Smith, B.W., 1977, ApJS, 35, 419

Roettiger, K., Loken, C. \& Burns, J.O., 1997, ApJSS, 109, 307

Rothschild, R.E., Baity, W.A., Marscher, A.P. \& Wheaton, W.A., 1981, ApJL, 243, L9

Schindler, S. \& Müller, E., 1993, A\&A, 272, 137

Schombert, J.M., 1987, ApJS, 64, 643

Schwarz, R.A., Edge, A.C., Voges, W., Böhringer, H., Ebeling, H. \& Briel, U.G., 1992, A\&A, 256, L11

Slezak, E., Durret, F. \& Gerbal, D., 1994, AJ, 108, 1996

Snowden, S.L., McCammon, D., Burrows, D.N. \& Mendenhall, J.A., 1994, ApJ, 424, 714

Snyder, W.A., Kowalski, M.P., Cruddace, R.G., Fritz, G.G., Mid- dleditch, J., Fenimore, E.E., Ulmer, M.P. \& Majewski, S.R., 1990, ApJ, 365, 460

Thomas, P.A. et al., 1998, MNRAS, 296, 1061

White, D.A., Fabian, A.C., Allen, S.W., Edge, A.C., Crawford, C.S., Johnstone, R.M., Stewart, G.C. \& Voges, W., 1994, MNRAS, 269, 589

White, D.A. \& Fabian, A.C., 1995, MNRAS, 273, 72

White, D.A., Forman, W. \& Jones, C., 1997, MNRAS, 292, 419

White, S.D.M., Navarro, J.F., Evrard, A.E. \& Frenk, C.S., 1993, Nature, 366, 429 